\title{
Atmosphere-Ocean Interactions at Strong Couplings in a Simple Model of El Niño
}

\author{
A. NAVARRA \\ Centro Euromediterraneo sui Cambiamenti Climatici, and Istituto Nazionale di Geofisica e Vulcanologia, Bologna, Italy \\ J. TRIBBIA \\ National Center for Atmospheric Research,* Boulder, Colorado \\ G. CONTI \\ Centro Euromediterraneo per i Cambiamenti Climatici, Bologna, Italy
}

(Manuscript received 30 October 2012, in final form 5 July 2013)

\begin{abstract}
The understanding of the dynamics of the El Niño-La Niña phenomenon in the tropical Pacific has been the subject of an impressive number of works in the last 20 years. The delayed oscillator theory provides an interpretative framework that has allowed enormous advances in understanding. Much evidence that stochastic forcing does play a role in the dynamics of ENSO has been discussed and it is possible to shape a theory of El Niño as a stochastically forced linear system. However, it is still uncertain if El Niño is a self-sustained nonlinear oscillatory system, a chaotic system, or a stochastically forced linear system. The authors propose in this paper that it is possible to have realistic El Niño probability distributions assuming that the system is a nonlinear stochastically forced system. In this paper a simple system is proposed that retains the main characteristics of the El Niño-La Niña variations, such as the skewness and the autocorrelation, and it is shown how solutions for the probability distribution can be obtained using a Fokker-Planck equation.
\end{abstract}

\section{Introduction}

Simple models of El Niño-Southern Oscillation (ENSO) were able to reproduce the basic characteristic behavior and scale of ENSO (Cane and Zebiak 1985; Cane et al. 1986; Zebiak and Cane 1987) and a dynamical framework was developed to explain the underlying dynamics in terms of delayed oscillators (Schopf and Suarez 1988; Suarez and Schopf 1988; Battisti and Hirst 1989; Philander 1990; Jin and Neelin 1993; Neelin et al. 1994, 1998) or in terms of the recharge mechanism (Jin 1996, 1997). Other conceptual models have also been proposed, like for instance the advective-reflective oscillator (Picaut et al. 1997), but they have been shown to be an equivalent

\footnotetext{
* The National Center for Atmospheric Research is supported by the National Science Foundation.

Corresponding author address: Antonio Navarra, Viale Aldo Moro 44, 40129 Bologna, Italy.

E-mail: antonio.navarra@cmcc.it
}

representation of a more general oscillatory dynamics (Wang 2001).

However, the observations show that the time series of the ENSO indices is strongly irregular and the differentiation from one event to the next is strong, with significant differences in the termination and startup of the different phases. These observations prompted the hypothesis that ENSO could instead be described as a stochastically forced linear system (Penland and Sardeshmukh 1995; Moore and Kleeman 1999a,b; Thompson and Battisti 2000, 2001; Penland 1996). It is still unclear if the nature of ENSO is therefore a deterministic system with strong nonlinearities or a linear system stochastically forced (Chang et al. 1996; Philander and Fedorov 2003).

The issue of the statistical distribution of the ENSO phenomenon has been considered several times. Early works (Burgers and Stephenson 1999) showed that there is considerable deviation from normality in the west and east Pacific, but some noticeable deviations exist also in the central Pacific region where widely used indexes such as ENSO3 and ENSO3.4 are computed. They also 
showed that histograms are suitable to identify deviations from normality and to estimate the probability distribution of the SST in each point or region. Monahan and Dai (2004) investigated the non-Gaussian character of ENSO using principal component analysis. The skewness has been attributed to nonlinear interactions by An et al. (2005), showing that probably a clear distinction between the previous choices may not be necessary and the final conclusion is still unclear (Kapur et al. 2012).

A simple linear model proposed by Jin (1997) can sustain oscillations as a function of the interaction parameter between the wind stress and the SST. A single oscillatory mode exists for most of the range of the parameter except for regions of large values, corresponding to a very tight coupling between SST and wind stress, and also for very small values, corresponding to relative independence. The model shows oscillations also when a cubic nonlinear term is introduced following Suarez and Schopf (1988). Jin concentrated on the oscillatory range to develop further his theory deeming unphysical both weak and strong coupling since they could not sustain oscillations. Available evidence indicates that ENSO is indeed coupled, so it is reasonable to ignore the weak coupling limit, but the high end of the parameter range (strong coupling) is more puzzling. Strong dynamical constraints are required to prevent the system to exit the range of values for which oscillations exist, but no explanation has been put forward. On the other hand, the estimation of the interaction coefficient has proven elusive. It is difficult to reduce the complex relation between wind and SST to a single simple parameter that could be diagnosed from observation and models. It is possible that energy-based approaches (Fedorov et al. 2003; Brown and Fedorov 2010) can shed some light on the issue, but they have still to deliver a definitive answer that would allow us to assess the interaction regime for observations or the models.

There is, however, a third possibility. It is possible that the coupled system is not so finely tuned, but it goes through different stages as the evolution of the basic state on longer time scales, like interdecadal variation and the interaction with anthropogenic climate change, progresses. It would be then conceivable that that there will be phases of strong interaction and phases of moderate interaction and so it is worthwhile to further investigate the strong interaction phase. In this phase the system does not have oscillations, but it can have a number of stable or unstable critical points and in the case of Jin model it will have two stable points. Transitions from one point to the other are impossible in the deterministic dynamical system, but become possible if we introduce a stochastic forcing that allows fluctuations between two different states. In this theory what we call ENSO events are the transitions between two different states driven by noise. We will develop in this paper a theory of a stochastic ENSO showing that we can get realistic probability distributions and that we can identify different ENSO states in the observations. A suitable equation for the evolution of the probability distribution will be developed. In this sense we are proposing that ENSO is a stochastically driven system with strong nonlinearities.

Although we are using additive noise as the stochastic component, we do not advocate it as the only forcing mechanism for ENSO. Most probably multiplicative noise will need to be considered (Penland and Sardeshmukh 1995; Penland 2003; Sura et al. 2005; Berner 2005), but the framework that we introduce here is not limited to additive noise and it can be extended to consider other kind of noises. It is a framework that allows a theoretical investigation of various kind of noise, additive, multiplicative, or colored, that can be involved in developing a full theory of ENSO. We show that even a drastically simplified model has some of the features found in the behavior of a Niño index.

The asymmetry of the positive and negative ENSO events has also been discussed by several studies, although the issue has often been discussed together with the irregularity of the oscillations. Jin et al. $(1994,1996)$ and Tziperman et al. (1994) proposed that the interactions of the seasonal cycle with the chaotic dynamics of the system caused the irregularity, whereas Penland and Sardeshmukh (1995), Moore and Kleeman (1999a,b), Kleeman (2008), and Thompson and Battisti (2000) discussed the effect of stochastic forcing onto the nonnormal modes of the linear system. The role played by the nonlinear component of the thermal advection has been investigated by Jin et al. (2003) and An and Jin (2004). Timmermann et al. (2003) proposed an intermittent nonlinear theory and Majda et al. (2006) investigated stochastic effects in deterministic systems. Other nonlinear mechanisms that generate an asymmetry between El Niño and La Niña cases have been proposed, such as vertical mixing processes (Wang and McPhaden 2000) and asymmetric atmospheric responses (Kang and Kug 2002).

Recently, some interest has arisen as to the interaction of the Madden-Julian oscillation (MJO; Madden and Julian 1971) with ENSO (Kapur et al. 2012). The MJO can contribute to the generation of positive anomalies in the east and central Pacific (Zhang and Gottschalck 2002; Zhang 2001) and models tend to have a better representation of ENSO events if MJO variability is enhanced or better represented (Zavala-Garay et al. 2008; Lengaigne et al. 2004). Correlations between ENSO and MJO activity have been detected in the observations (Zhang and Gottschalck 2002; Hendon et al. 2007), 
whereas models have advanced significantly the capability to describe the MJO (Subramanian et al. 2011; Sperber et al. 2005; Gualdi et al. 1997) and recently models have shown a capacity to improve both the MJO and ENSO (Neale et al. 2008). This correlation has led to the consideration of multiplicative stochastic noise as a generalization of the additive noise more generally used. However, many of the techniques that we are going to describe are not dependent on the additive or multiplicative nature of the noise.

In this paper we are proposing a new approach to the ENSO variability that centers on the hypothesis that ENSO can be described as a nonlinear system that can sustain oscillations as a function of the coupling strength, but that can also generate fluctuations when oscillations cannot be sustained between different states via stochastic fluctuations. The probability distribution of the ENSO will be obtained directly via solving the FokkerPlanck equation of a simplified model and it will be shown that the asymmetry can be explained in terms of a simple parameter that can be interpreted as related to the average seasonal MJO activity. The general picture that emerges is that ENSO is acted on by different mechanisms as the coupling strength and intensity of the stochastic forcing vary, providing possible explanations for the variety of behavior that we experience in models and in the observations.

Although strict realism is not the purpose of this paper, we will be able to offer a new framework for investigating ENSO from a different angle and the model shows some interesting properties.

\section{The probability distribution of ENSO}

Considering the nonnormalized 1871-2011 Niño-3.4 index (available online at www.esrl.noaa.gov/psd/gcos_ wgsp/Timeseries/Nino34) computed from version 1 of the Hadley Centre Sea Ice and Sea Surface Temperature dataset (HadISST1; Rayner et al. 2003) as the area averaged over $5^{\circ} \mathrm{S}-5^{\circ} \mathrm{N}$ and $170^{\circ}-120^{\circ} \mathrm{W}$, we can estimate the stationary component of the empirical probability distribution by computing the histogram of the Niño-3.4 anomalies. A 5-month running mean to remove the highfrequency components has been applied. The estimated probability distribution is an asymmetric shape with a marked shift toward negative anomalies that generates a peak around -0.2 . A good fit can be obtained with a superposition of two normal distributions, suitably weighted,

$$
P(x)=p \exp \left[-\frac{\left(x-x_{1}\right)^{2}}{s_{1}^{2}}\right]+(1-p) \exp \left[-\frac{\left(x-x_{2}\right)^{2}}{s_{2}^{2}}\right],
$$

with the mixing parameter $p$ constrained as $0<p<1$, resulting in the superposed line visible in Fig. 1. The bottom panel shows also the same fit applied to the residual obtained subtracting the filtered components from the total anomalies. In this case there is no sign of a double Gaussian nature and a single Gaussian is a good fit. This result suggests that variability faster than 5 months may be described by Gaussian stochastic fluctuations, whereas the slower variability is well captured by two states represented by the Gaussians.

However, the models tell a different story. Just to give an appreciation of the variability that different models can exhibit, we show in the following figures the estimated probability distribution obtained from long simulations of the Centro Euromediterraneo sui Cambiamenti Climatici (CMCC) Scale Interaction Experiment (SINTEX) model (Navarra et al. 2008; Gualdi et al. 2003). In these experiments a different resolution atmospheric model was coupled to the same ocean model. It is a T106 spectral resolution for Fig. 2 and spectral T30 for Fig. 3. It is possible to note that the T106 model shows some signs of developing an asymmetry like the observations whereas the T30 model appears to be rather symmetric, resembling a pure normal distribution.

The results can also be understood in terms of the two Gaussians composing the probability distribution. If we plot the individual states, combined with the weights obtained from the fit, we get Fig. 4. The probability of a particular observed value can therefore be understood as the probability of being in one state or the other. It is interesting to note that the variability in the T30 case is so poor that the second state is almost disappearing, indicating that in fact there is little difference from a simple normal distribution with zero mean in this case.

We can shed some light on the interpretation of these results by considering some simple idealized cases. Let us consider the case of an oscillation on which some Gaussian white noise with zero mean is added (top panel, Fig. 5). The time series of the oscillation shows variability, but the probability histogram has a noticeable two-peak structure that can be readily identified as two separate states. However, the states are symmetric, both in the location of their maximum and in the amount of probability they explain. The two states are equally probable. The result is rather insensitive to the amount of noise, as the histogram in this case easily captures the signature of the underlying oscillation even in the case of strong noise (not shown). As the noise becomes dominant the probability distribution loses track of the oscillation, showing only the Gaussian distribution of the noise. The result is rather insensitive to the particular shape of the oscillation; a square wave yields the same symmetric two-peak result, with a better separation 

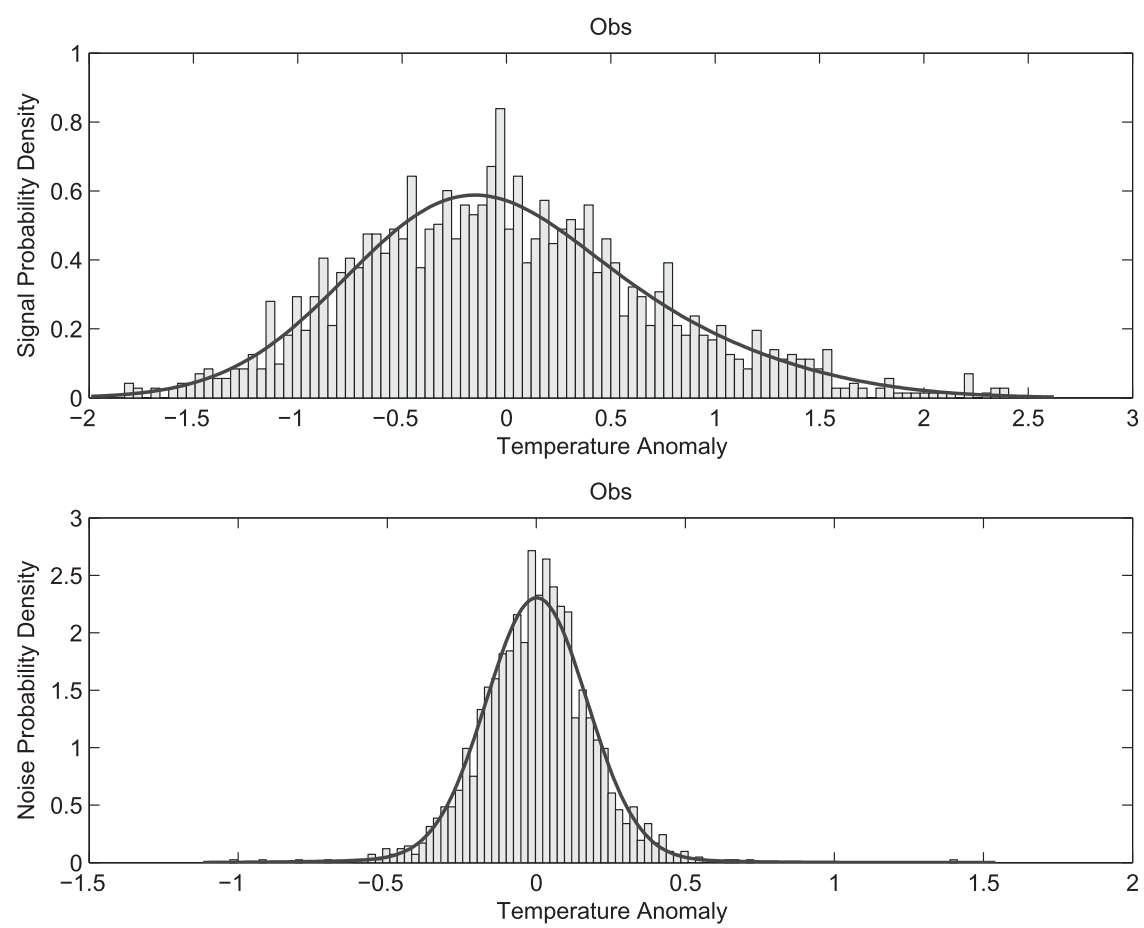

FIG. 1. The estimated probability distribution of the Niño-3.4 index for observations. The monthly mean Niño-3.4 data have been obtained from the Climate Prediction Center (CPC) for the period 1871-2012, the seasonal cycle has been removed, and a 5-month running mean has been applied. (top) The PDF of the filtered data estimated as a normalized histogram. (bottom) The same estimate applied to the residual signal after the running mean has been subtracted. In both panels, the line represents the best fit of a mixture of two normal distributions. The deviations from the running mean appear to be represented well by a single normal distribution. The parameters of the fit have been determined at $99 \%$ confidence level.

between the peaks due to the faster transition between high and low states.

The only way to get an asymmetric distribution like in the observations, within the assumptions of additive Gaussian white noise and a two-state ENSO, is to allow different resident times in the two different states. Figure 6 shows the same information as Fig. 5, but in this case the noise has been superposed onto a square wave with different lengths of the extreme states. We can see how the asymmetry can be easily identified and the relative weight of the states found. The probability distribution signature of a pure oscillation can also easily be identified, even if there is a considerable amount of noise superposed. The observation seems to indicate that the asymmetry is intrinsic and cannot be explained with stochastic forcing.

Visual inspection of the T30 time series will not give the same impression and also a false impression can be obtained from spectral analysis of the same experiments (Navarra et al. 2008) that yields peaks with some significance. In reality there are almost no oscillations in the case of the T30. This simple diagnostic is also showing a very powerful method to evaluate the quality of the performance of models.

\section{An equation for the probability distribution}

We will describe in this section how we can formulate an equation that will allow us to investigate the properties of the probability distribution itself. In general terms a system describing ENSO and an additive stochastic forcing can be written as a Langevin-like equation:

$$
\dot{x}_{\mu}(t)=f_{\mu}[\mathbf{x}(t)]+\sqrt{Q} \epsilon_{\mu}(t),
$$

where $\mathbf{x}(t)=\left[x_{1}(t), \ldots, x_{K}(t)\right]$ represents the instantaneous state of the system and $f_{\mu}(\mathbf{x})$ a differentiable function of $\mathbf{x}$ for $\mu=1, \ldots, K$. The noise functions $\epsilon_{\mu}(t)$ are defined by their correlation functions as

$$
\gamma_{\mu \nu}\left(t, t^{\prime}\right)=\left\langle\epsilon_{\mu}(t) \epsilon_{\nu}\left(t^{\prime}\right)\right\rangle_{\epsilon}=2 Q \delta_{\mu \nu} \delta\left(t-t^{\prime}\right)
$$



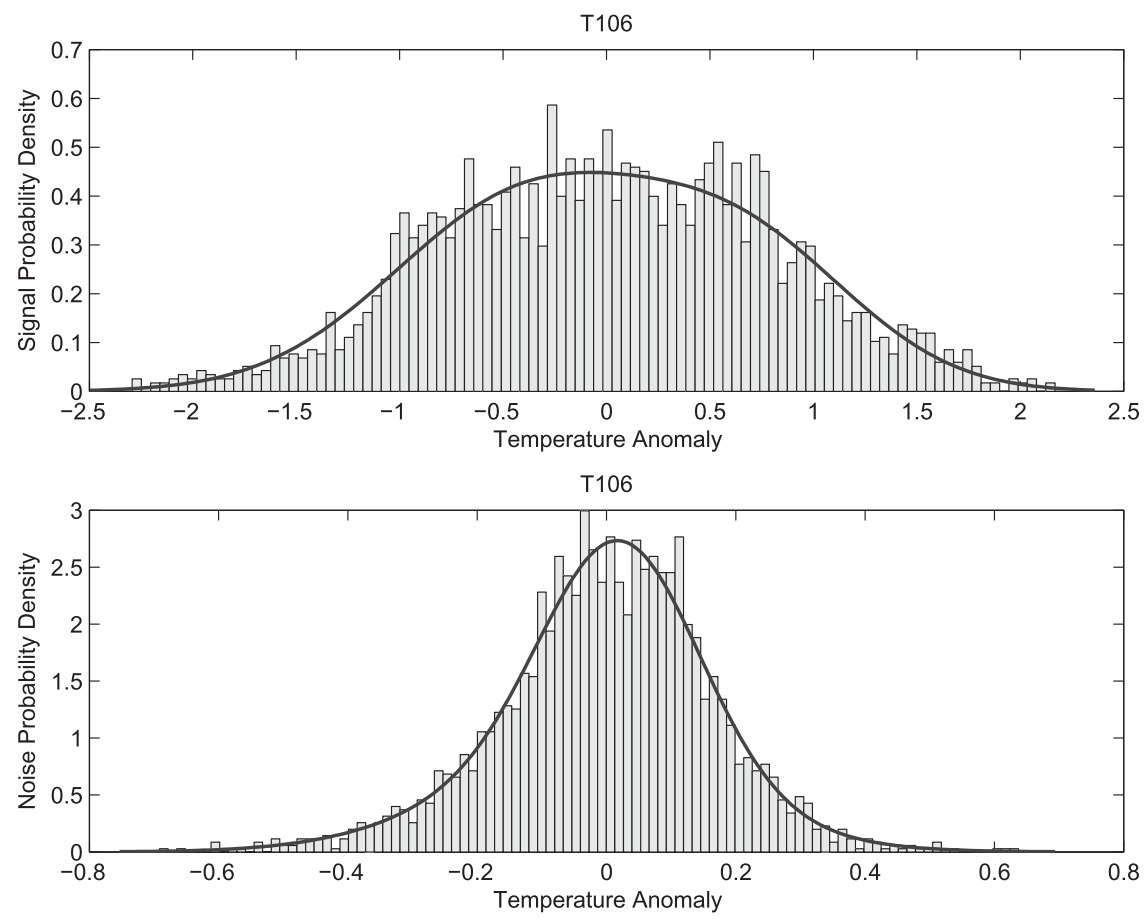

FIG. 2. As in Fig. 1, but for the monthly mean Niño-3 index obtained from a 200-yr simulations of the CMCC coupled spectral T106 model (Navarra et al. 2008; Gualdi et al. 2003).

and they have zero means; $\langle.\rangle_{\epsilon}$ is an average with respect to the probability distribution of the realizations of the stochastic variables $\epsilon_{\mu}(t)$.

\section{a. The Fokker-Planck equation}

We are interested in the transition probability distribution for the stochastic process $P\left(\mathbf{x}, t, \mathbf{x}_{0}, t_{0}\right)$, describing the probability for the system to be in $\mathbf{x}$ at time $t$, given that it was in $\mathbf{x}_{0}$ at time $t_{0}$. In what follows we shall often omit the dependence on the initial data and use the simplified notation $P(\mathbf{x}, t)$. Using the Gaussian nature of the noise it is possible to write a Fokker-Planck equation (FP) for $P(\mathbf{x}, t)$ (Risken 1989) with an implied summation over repeated indices:

$$
\frac{\partial P(\mathbf{x}, t)}{\partial t}=\frac{\partial}{\partial x_{\mu}}\left[Q \frac{\partial P(\mathbf{x}, t)}{\partial x_{\mu}}-f_{\mu}(\mathbf{x}) P(\mathbf{x}, t)\right] .
$$

The Fokker-Planck equation can be written also for multiplicative noise, but we will consider only the additive case here; $Q$ represents here a constant diffusion coefficient while $f_{\mu}$ is the drift term. In principle, the solution of this equation contains all the information we need on the probability distribution and its evolution in time, but explicit solution can often be very difficult except in simple cases. However, we might see the situation from a different angle by realizing that the solution of this equation can be written as a path integral (Haken 1976; Navarra et al. 2013)

$$
P(\mathbf{x}, t)=\int[\mathcal{D} \mathbf{x}(\tau)] \exp [-S(\mathbf{x})] P\left(\mathbf{x}, t_{0}\right)
$$

where the integration is done over all paths $\mathbf{x}(t)$ that go from an initial state $x_{0}$ at time $t_{0}$ to a final state $x_{f}$ at time $t_{f}$. The functional $S(\mathbf{x})$ is the continuous OnsagerMachlup action that can then be defined in the white noise case as

$S(\mathbf{x})=\frac{1}{4 Q} \int_{0}^{t_{f}}\left[\left[\dot{x}_{\mu}-f_{\mu}(\mathbf{x})\right] \delta_{\mu \nu}\left[\dot{x}_{\nu}-f_{\nu}(\mathbf{x})\right]+2 Q \frac{\partial f_{\mu}}{\partial x_{\mu}}\right] d t$.

The extra divergence term in the action is generated by the difficulty of defining the derivative of a stochastic process and it corresponds to the choice of the Stratonovich differentiation for the stochastic process. It is worthwhile to mention here that the introduction of noise into a differential equation changes profoundly the character of the equation. In particular, individual realizations have little meaning and it opens a classification between quantities that can be measured in a repeatable and consistent manner (observables) and quantities that cannot really be measured. 

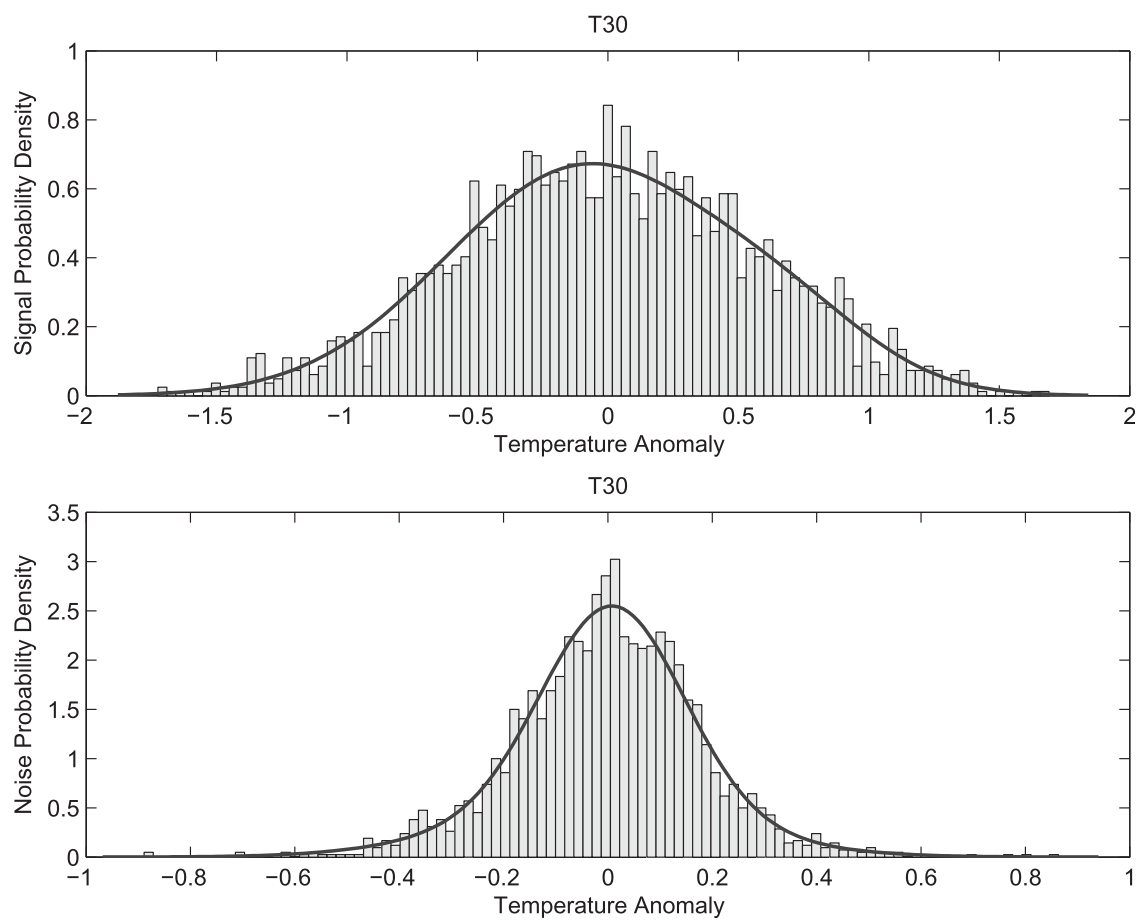

FIG. 3. As in Fig. 1, but for the monthly mean Niño-3.4 index obtained from a 200-yr simulations of the CMCC coupled spectral T30 model (Navarra et al. 2008; Gualdi et al. 2003).

Equation (4) can be solved by separating the time dependence and solving a stationary problem:

$$
P(x, t)=\sum_{n=0}^{\infty} \exp \left(-\lambda_{n} t\right) \phi_{n}(x, t)
$$

where the $\phi$ are the eigenfunctions and eigenvalues of the Fokker-Planck stationary operator

$$
L_{\mathrm{FP}}=\frac{\partial}{\partial x_{\mu}} Q \frac{\partial}{\partial x_{\mu}}-\frac{\partial}{\partial x_{\mu}}\left[f_{\mu}(\mathbf{x})(.)\right] .
$$

Note that $L_{\mathrm{FP}}$ is not self-adjoint and therefore we would need to consider also the eigenvectors of its adjoint. However, a self-adjoint system can be obtained if the drift term can be expressed in terms of a drift potential $U(x, t)$ according to $f_{\mu}=-\left[\partial U(x, t) / \partial x_{\mu}\right]$. In this case it is then possible to use the transformation

$$
P(x, t)=\exp \left[-\frac{U(x)}{2 Q}\right] \psi(x, t),
$$

obtaining

$$
\frac{\partial \psi}{\partial t}=L \psi=Q \frac{\partial^{2} \psi}{\partial x^{2}}-V(x) \psi
$$

where

$$
V(x)=\frac{1}{4 Q}\left(\frac{\partial U(x)}{\partial x}\right)^{2}-\frac{1}{2} \frac{\partial^{2} U(x)}{\partial x^{2}}
$$

The operator $L$ is now self-adjoint and it has the same eigenvalues as $L_{\mathrm{FP}}$. Their eigenfunctions are linked so that

$$
\phi_{n}(x)=\exp \left[-\frac{U(x)}{2 Q}\right] \psi_{n}(x)
$$

and they form an orthonormal system such that

$$
\int \psi_{n} \psi_{m} d x=\int \exp \left[\frac{U(x)}{Q}\right] \phi_{n} \phi_{m}=\delta_{m n}
$$

The operator $L$ can be expressed in a compact way as

$$
L=-\hat{a} a,
$$

where

$$
a=\sqrt{Q} \exp \left(-\frac{U}{2 Q}\right) \frac{\partial}{\partial x} \exp \left(\frac{U}{2 Q}\right) \text { and }
$$

$$
\hat{a}=-\sqrt{Q} \exp \left(\frac{U}{2 Q}\right) \frac{\partial}{\partial x} \exp \left(-\frac{U}{2 Q}\right) .
$$

The existence of stationary solutions depends on the asymptotic behavior of the potential $V(x)$. For natural 

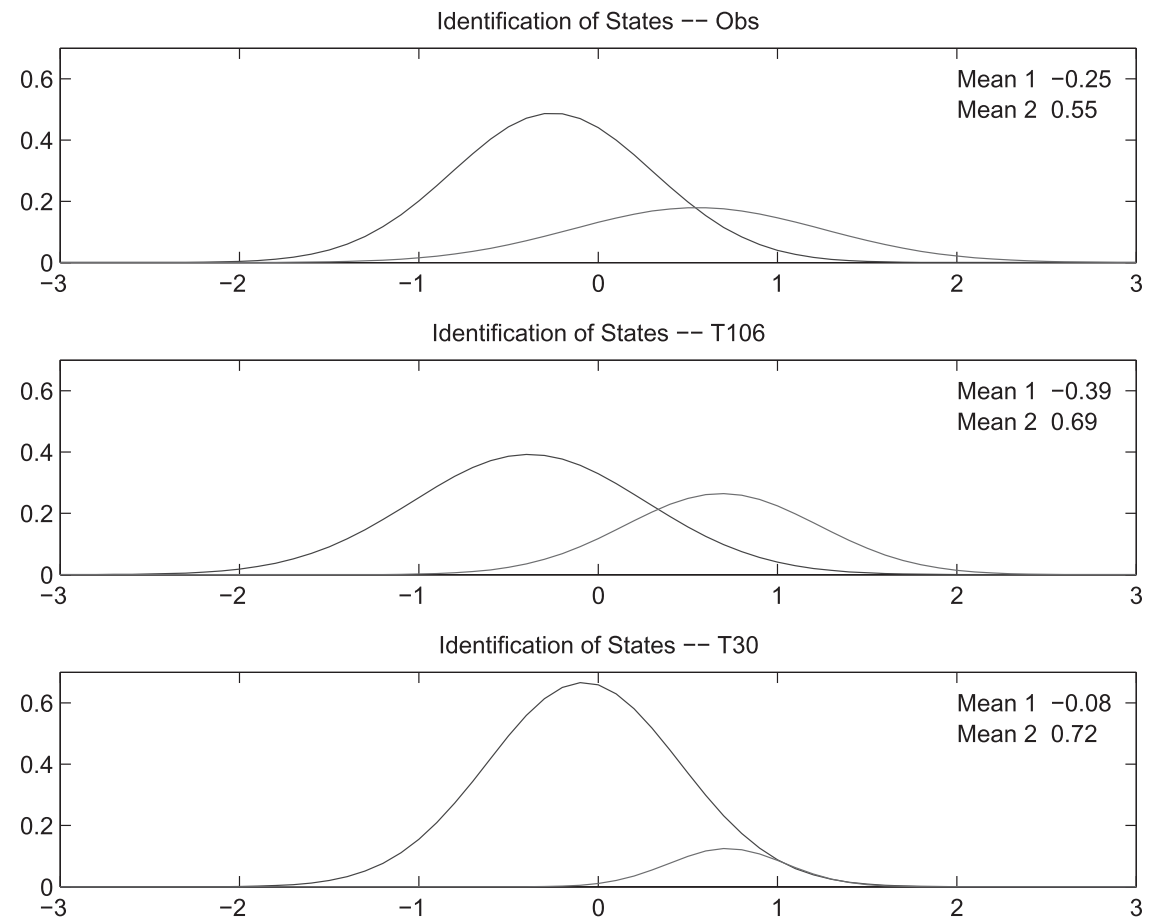

FIG. 4. Representation of the two normal distributions entering the best fit in the previous pictures. The Gaussians are well separated and they can be interpreted as possible states of the system. The pictures show estimated states for (top) observations and the (middle) T106 and (bottom) T30 models. We can interpret the warm state as El Niño and the cold state as La Niña.

boundary conditions where the potential goes to very large values for $x \rightarrow \infty$ the operator $\hat{a}$ becomes the adjoint of $a$ and stationary states exist. However, also in the opposite case when the potential becomes very negative it is possible to find an eigenfunction and eigenvalues.

Defining the eigenfunction and eigenvalues of $L$ as

$$
L \psi_{n}=-\lambda \psi_{n},
$$

we can write the transition probability as

$$
P\left(x, t \mid x^{\prime} t^{\prime}\right)=e^{\left[U(x)-U\left(x^{\prime}\right)\right] / 2 Q} \sum_{n} \psi_{n}(x) \psi_{n}\left(x^{\prime}\right) e^{-\lambda_{n}\left(t-t^{\prime}\right)} .
$$

The transition probability allows us to compute all the joint probability distributions that express the probability $W_{n}$ of the system assuming the values $\left(x_{1}, t_{1}\right)$, $\left(x_{2}, t_{2}\right), \ldots,\left(x_{n}, t_{n}\right)$. There is an infinite hierarchy of joint distribution factors that collectively defines the property of the stochastic system; however, for a Markov system all the higher-order $W$ can be obtained from the twopoint distribution $W_{2}$ that describes completely the system at equilibrium according to

$$
W_{2}\left(x, t \mid x^{\prime}, t^{\prime}\right)=P\left(x, t \mid x^{\prime}, t^{\prime}\right) W_{\mathrm{st}}\left(x^{\prime}\right) \quad t \geq t^{\prime},
$$

where $W_{\text {st }}$ is the stationary distribution, corresponding to the eigenvalue zero. It is easy to show that a solution of Eq. (8) for the eigenvalue zero is

$$
W_{\text {st }}(x)=\exp \left[-\frac{U(x)}{2 Q}\right] .
$$

However, such a solution exists only for suitable boundary conditions. The so-called natural boundary conditions correspond to the potential going to very large values as $|x| \rightarrow \infty$, reflecting boundary conditions requiring $\psi \rightarrow 0$ can then guarantee the existence of a stationary solution.

In terms of eigenfunctions and using the explicit form of the stationary solution we get

$$
W_{2}\left(x, t \mid x^{\prime} t^{\prime}\right)=e^{\left(U(x)+U\left(x^{\prime}\right)\right) / 2 Q} \sum_{n} \psi_{n}(x) \psi_{n}\left(x^{\prime}\right) e^{-\lambda_{n}\left(t-t^{\prime}\right)}
$$

and the correlation function is therefore given by the expectation value

$\langle x(t+\tau) x(t)\rangle=\iint x x^{\prime} W_{2}\left(x, t+\tau \mid x^{\prime}, t\right) d x d x^{\prime}$.

The expectation value of any function $g(x, t)$ can be readily calculated as follows: 

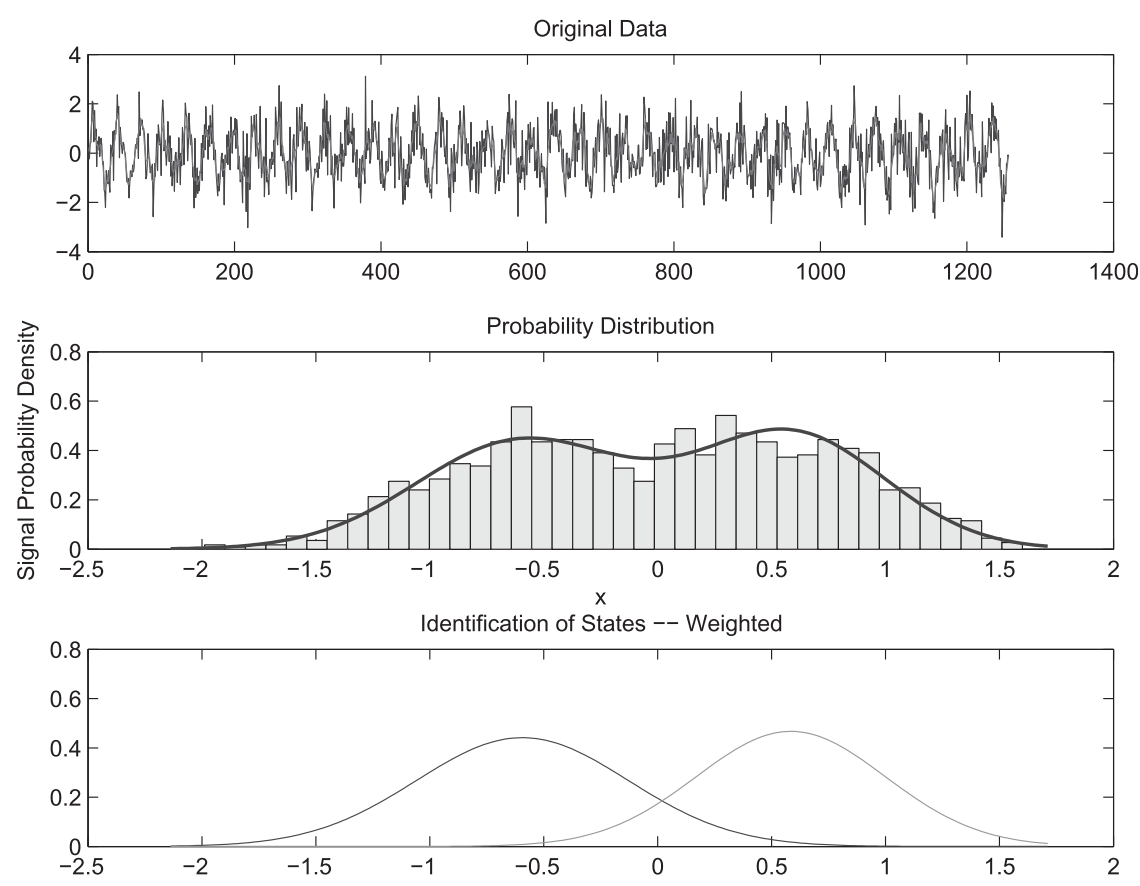

FIG. 5. A time series for sinusoidal oscillations with noise (top) for an arbitrary frequency and unit amplitude. (middle) The estimated probability distribution clearly shows two peaks (bottom) that can be readily identified as two symmetric, equal-amplitude states.

$$
\langle g(x, t)\rangle=\int_{-\infty}^{\infty} g(x, t) P(x, t) d x ;
$$

in a similar way one can obtain $\langle f(x)\rangle$, simply integrating over time.

\section{$b$. The relation with the Schrödinger equation}

The modified form of the Fokker-Planck equation (10) is suggestive of a possible link to other applications in physics. It closely resembles the Schrödinger equation
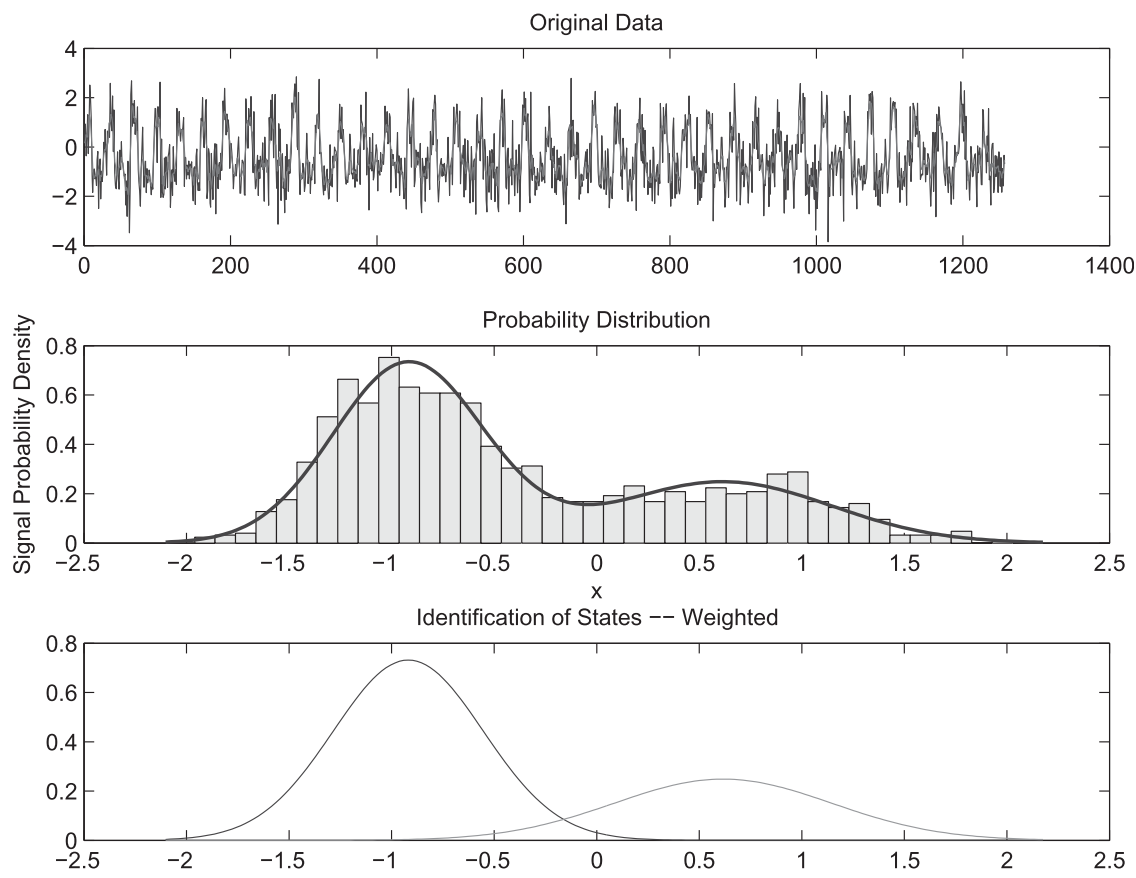

FIG. 6. As in Fig. 5, but for the case of a square wave of the same frequency and amplitude, but with different staying time for the positive and negative values. 


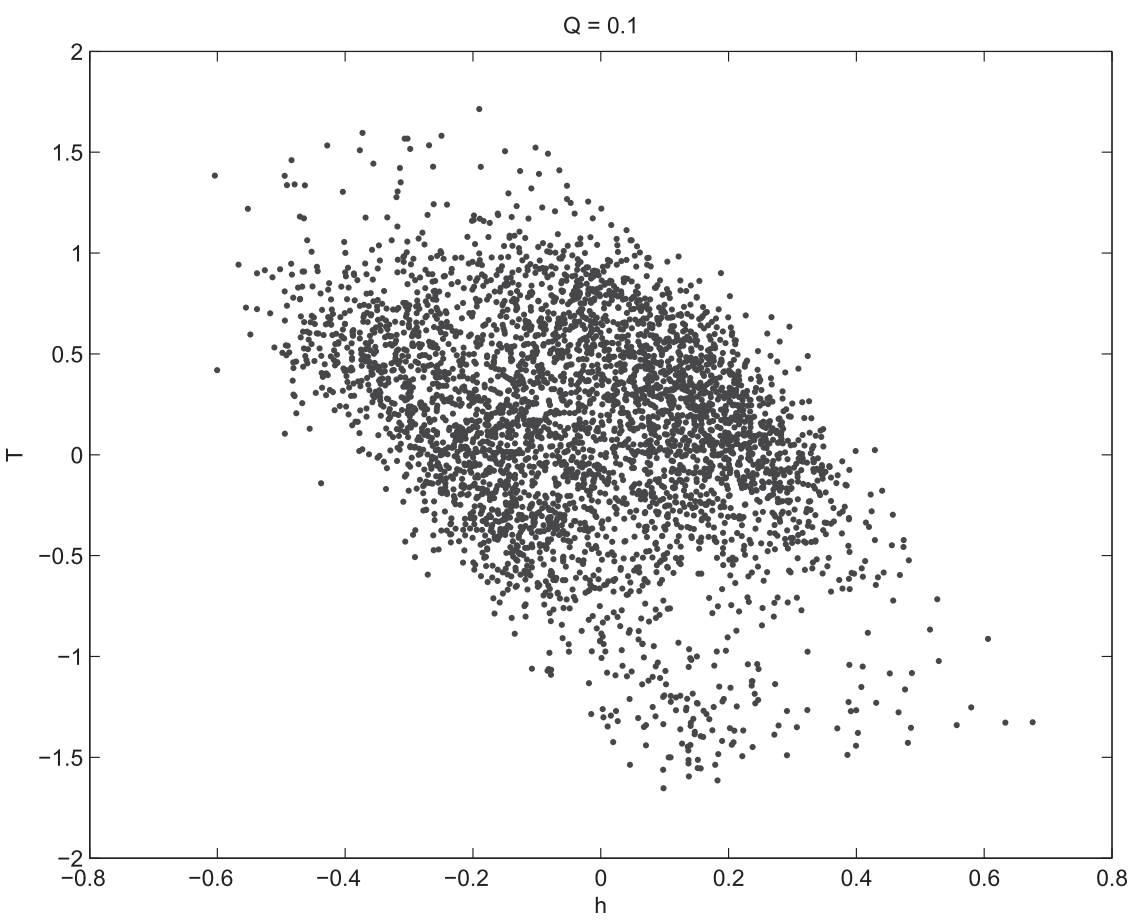

FIG. 7. Trajectory plot of a solution from (39) considering noise; $Q=0.1, \mu=21 / 15$.

used in quantum mechanics and this observation may be worth investigating a little bit more since both equations describe the evolution of probability or of quantities closely related to it. There is also another reason to look further in this relation. There are an enormous number of methods developed to deal with problems involving the Schrödinger equation and a multidecadal expertise in discussing the concepts and interpretations required. The potential of trying to use some of this wealth for application in our field is simply too large to ignore.

The detail of the relation linking the two equations can be made explicit. The Schrödinger equation for a point particle of mass $m$ can be written as

$$
i \hbar \frac{\partial \psi_{S}}{\partial t}=\frac{-\hbar^{2}}{2 m} \frac{\partial^{2} \psi_{S}}{\partial x^{2}}+V(x, t) \psi_{S}
$$

where $m$ is the mass of the particle, $\hbar$ is the Planck constant divided by $2 \pi$, and $\psi_{S}$ is the quantum wave function.

An analytical continuation of the time $t=-i \tau$ (sometimes known as Wick's rotation in the physics literature) will transform this form of the Schrödinger equation into

$$
\frac{\partial \psi_{S}(x, \tau)}{\partial \tau}=\frac{\hbar}{2 m} \frac{\partial^{2} \psi_{S}(x, \tau)}{\partial x^{2}}-\frac{1}{\hbar} V(x, \tau) \psi_{S}(x, \tau)
$$

We can recover (10) in form by substituting $\hbar / 2 m \rightarrow Q$ and rescaling the potential $V$ by $\hbar$. It is interesting to note that the stochastic parameter $Q$ plays the same role as Planck's constant $\hbar$. They are the controlling parameters of the uncertainty of the system. The limit $Q \rightarrow 0$ will yield the deterministic solution of the evolution equations in a similar sense that the limit $\hbar \rightarrow 0$ yields the classical (deterministic) solutions in the case of Schrödinger's equation.

The remaining difference is the interpretation of the wave function $\psi_{S}$. The probability distribution can be obtained from the solution of (24) by $P_{S}=\left|\psi_{S}\right|^{2}$ whereas the solution of the Fokker-Planck Eq. (10) is directly the probability distribution itself. Therefore, the evolution of the probability for a stochastic system is given by a Schrödinger-like equation, but whose solution $\psi$ represents the probability distribution itself (scaled by an exponential factor), rather than its density.

\section{A self-excitation ENSO model with a cubic nonlinear term}

A simple model of the ENSO system based on the recharge theory was proposed by Jin (1997). The model is a nonlinear system in terms of nondimensional variables $T, h ; T$ is representing the anomaly SST in the eastern Pacific, whereas $h$ is the anomaly of the depth of thermocline in the western Pacific. 


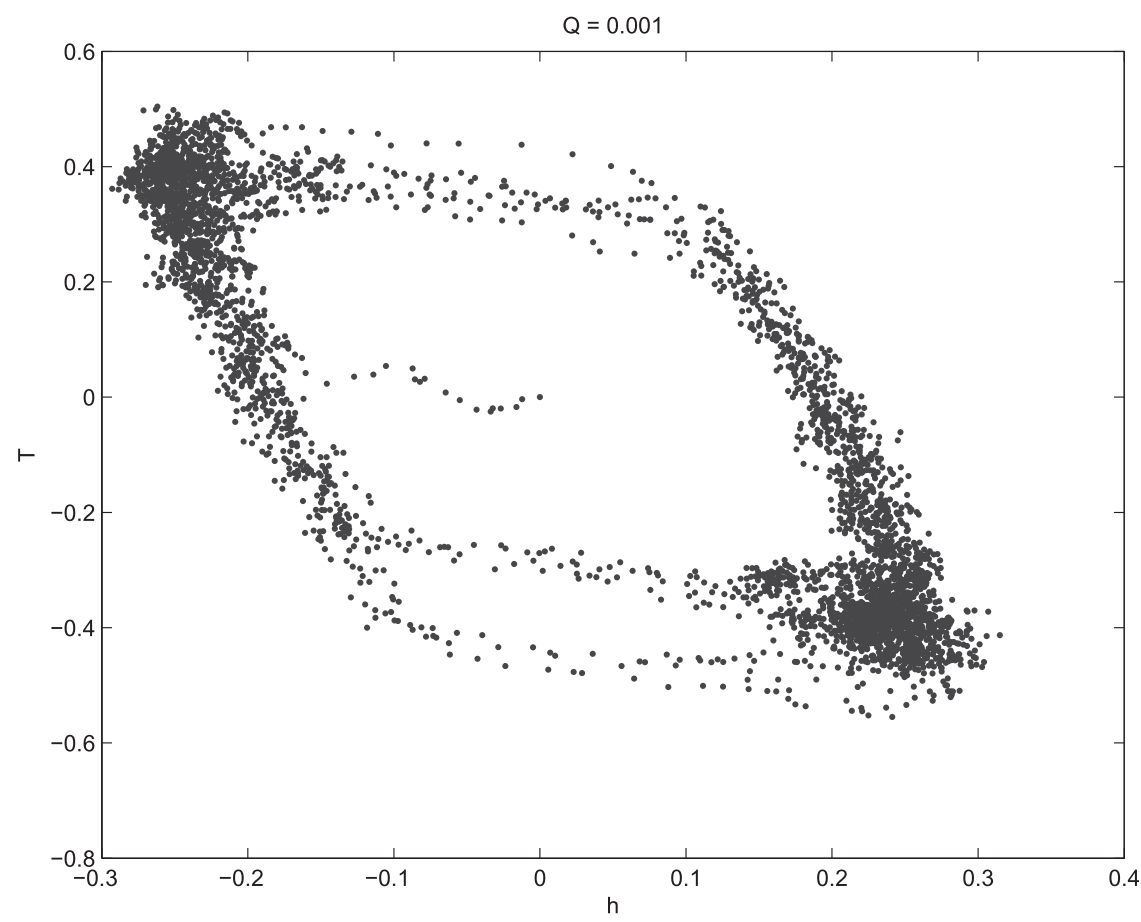

FIG. 8. Trajectory plot of a solution from (39) considering noise; $Q=0.001, \mu=21 / 15$.

$$
\begin{aligned}
& \frac{d h}{d t}=-r h-\alpha \mu b_{0} T, \\
& \frac{d T}{d t}=\left(\gamma \mu b_{0}-c\right) T+\gamma h-(b T+h)^{3} .
\end{aligned}
$$

The coupling strength will be measured by a parameter $\mu$ according to $\tau=b_{0} \mu T ; \mu$ measures the strength of the interaction between the SST and the wind stress relative to a scale $b_{0}$. The parameters can then be expressed as nondimensional values $c=1, \gamma=0.75, r=$ $0.25, \alpha=0.125$, and $b_{0}=2.5$ using the same choices of scales made by Jin (1997).

Jin (1997) studied the bifurcation diagram of the stationary solution of this system as a function of the interaction parameter $\mu$ and he found that the system has a single oscillatory solution for values of $\mu<16 / 15$ and then two exponential solutions that are stable for large values of $\mu>19 / 15$. The delayed oscillator theory focus on the parameter space below this threshold that allows oscillations and therefore the appearance of something like an ENSO cycle, but the introduction of stochastic noise also makes the regime with stable stationary states capable producing ENSO variability. They also investigated the behavior of the system under a stochastic forcing of strength $Q$, but still with a linear system to describe ENSO.

The addition of Gaussian white noise to the two equations in (39) will allow the system to vacillate between one stable point and the other. Figures 7 and 8 show the trajectory for increasing values of the noise strength $Q$. The corresponding probability distributions are peaked in correspondence of the stationary points (not shown). The fluctuation between the two stable states can be clearly seen and also the degradation of the bimodal nature of the distribution as the noise gets large, making the transition from one stable point to the other much easier. For larger values of the noise a completely normal distribution can be reached.

The variations of the trajectory are confined, however, and the path taken by the variables from one point to the other seems rather limited in a direction normal to the line joining the two centers. This suggests that if we could transform variables in such a way to introduce new variables $(\theta, z)$, obtained with a rotation

$$
\begin{aligned}
& h=z+\frac{0.8 \theta}{\mu}, \\
& T=\theta-\frac{0.8 z}{\mu},
\end{aligned}
$$

we will be able to transform the system in such a way that the stationary states in the $(z, \theta)$ coordinates are given by

$$
(0,0)\left(\frac{1}{2} \sqrt{\frac{15 \mu-16}{5}}, 0\right)\left(-\frac{1}{2} \sqrt{\frac{15 \mu-16}{5}}, 0\right)
$$




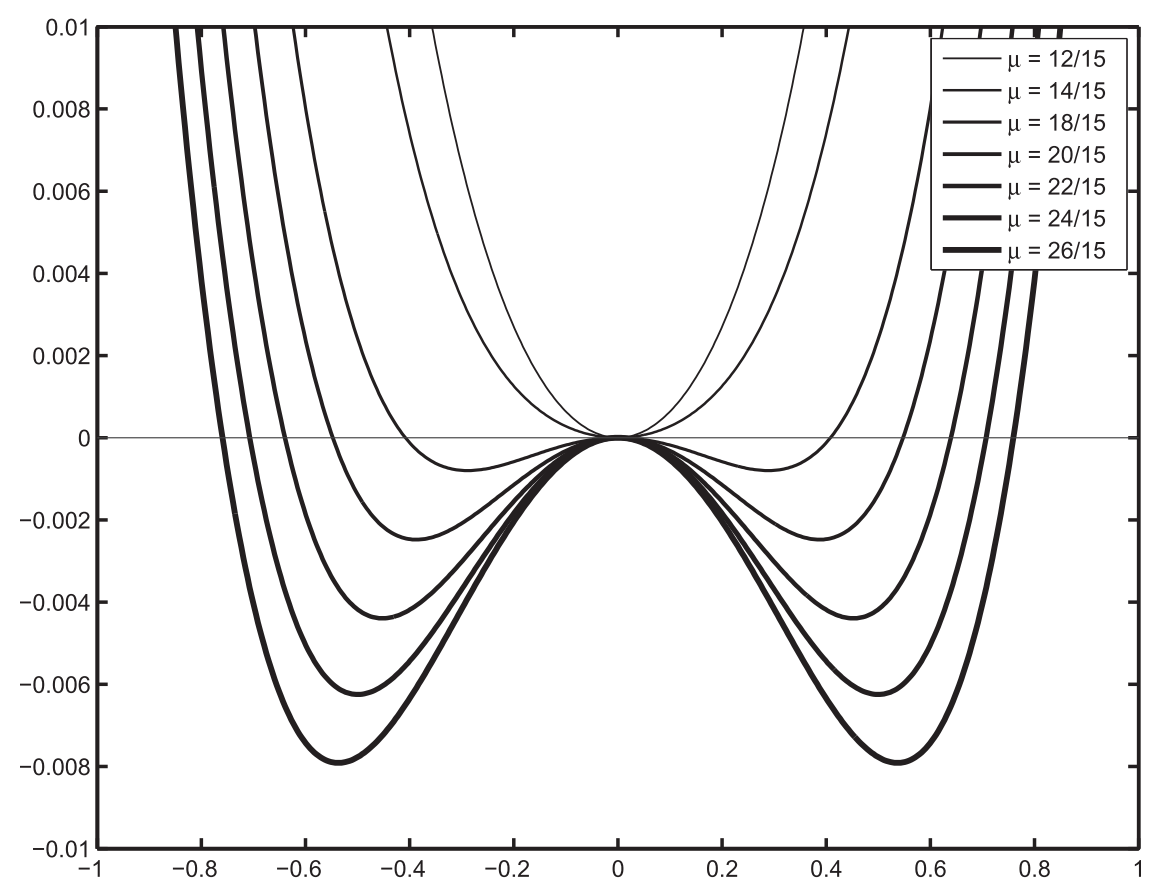

FIG. 9. The dependence of the potential of the one-dimensional model from the coupling parameter $\mu$. The symmetry is broken for $\mu=16 / 15$; for larger values the potential has two stable points in symmetric positions with respect to the ordinate axis. The separation increases with increasing values of the coupling.

along the $z$ axis. We can then transform the system to a single equation for $z$ by assuming that $\theta \approx 0$. This approximation implies that $h \approx z$ and $T \approx-\mu / 0.8 z$, which is consistent with the solution of the original system (39) as described in (Jin 1997).

We can now apply the arguments developed in section 3. Using the approximations just described we can get a one-variable stochastic equation:

$$
\dot{z}=f(z)+\sqrt{Q} \epsilon(t)
$$

with

$$
f(z)=\frac{z\left(-80 \mu z^{2}+60 \mu-64\right)}{100 \mu^{2}+64} .
$$

Defining the parameters

$$
\beta^{2}=\frac{60 \mu-64}{200 \mu^{2}+128} \quad \delta^{2}=\frac{40 \mu}{60 \mu-64},
$$

we can get a compact form

$$
f(z)=-4 \beta^{2} \delta^{2} z^{3}+2 \beta^{2} z .
$$

This forcing function $f(z)$ admits a potential $U(z)$ that can be obtained from $f(z)$ by integration,

$$
U(z)=-\int f(z) d z=\beta^{2} z^{2}\left(\delta^{2} z^{2}-1\right)
$$

and therefore we can use the self-adjoint form of the Fokker-Planck Eq. (10),

$$
V(x)=\frac{1}{4 Q}\left[\frac{\partial U(x)}{\partial x}\right]^{2}-\frac{1}{2} \frac{\partial^{2} U(x)}{\partial x^{2}}
$$

that in our case becomes

$$
V(z)=250\left[2 \beta^{2} \delta^{2} z^{3}+2 \beta^{2} z\left(\delta^{2} z^{2}-1\right)\right]^{2}+\beta^{2}-6 \beta^{2} \delta^{2} z^{2} .
$$

The complete Fokker-Planck equation for our simple model can then be written as

$$
\begin{aligned}
\frac{\partial \psi}{\partial t}= & Q \frac{\partial^{2} \psi}{\partial z^{2}}-\left\{250\left[2 \beta^{2} \delta^{2} z^{3}+2 \beta^{2} z\left(\delta^{2} z^{2}-1\right)\right]^{2}\right. \\
& \left.+\beta^{2}-6 \beta^{2} \delta^{2} z^{2}\right\} \psi
\end{aligned}
$$

The probability distribution can be obtained from the solutions of (36) via

$$
P(x, t)=\exp \left[-\frac{U(z)}{2 Q}\right] \psi(z, t) .
$$



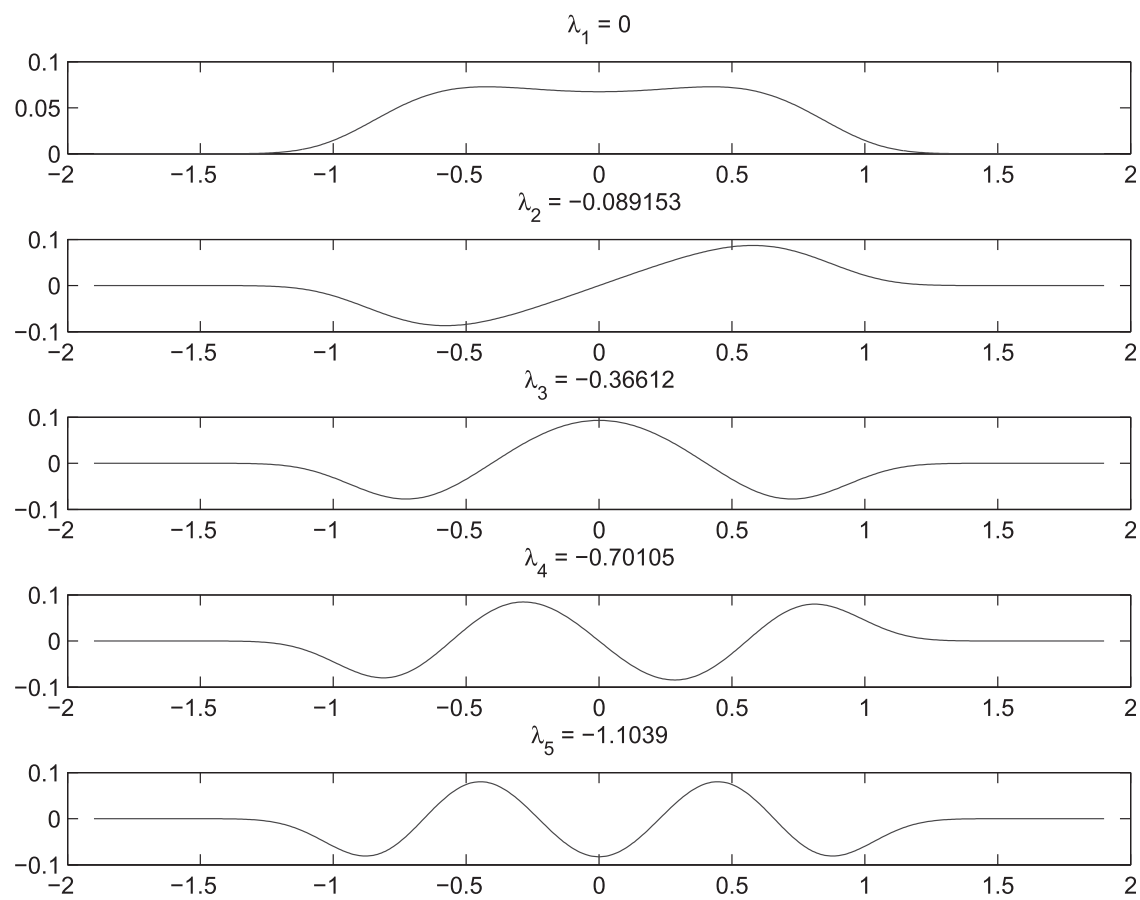

FIG. 10. The first five eigenfunction for the symmetric case. The first eigenfunction corresponds to the eigenvalue zero and therefore is the stationary distribution.

The one-dimensional system has an oscillatory solu- dependence of the potential on the coupling parameter is tion for $\mu<16 / 15$ and two stable solutions for $\mu>16 / 15$. There is also a critical point at $z=0$ that is stable for shown in Fig. 9. Crossing the threshold at 16/15 reveals a couple of symmetric stable points whose separation $\mu<16 / 15$ that becomes unstable for $\mu>16 / 15$. The increases with $\mu$. The depth of the two potential wells is
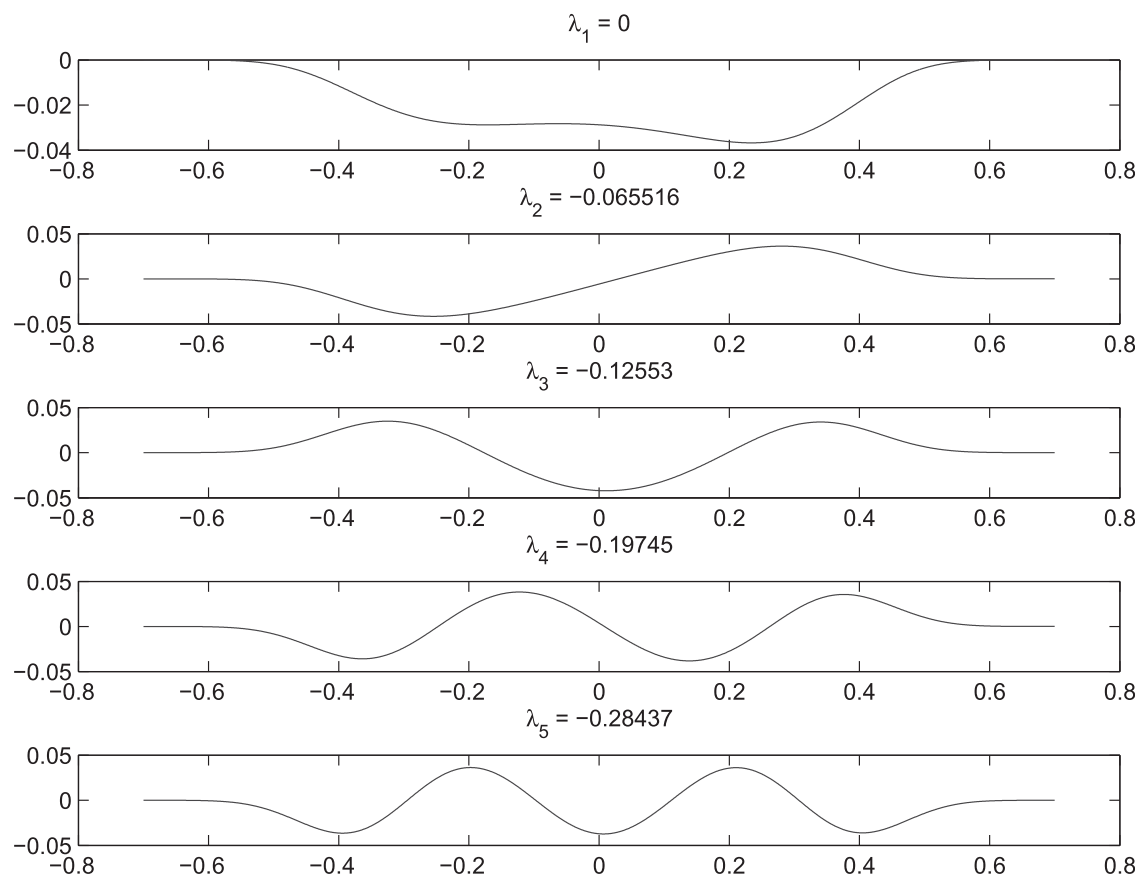

FIG. 11. As in Fig. 10, but for the asymmetric case. 


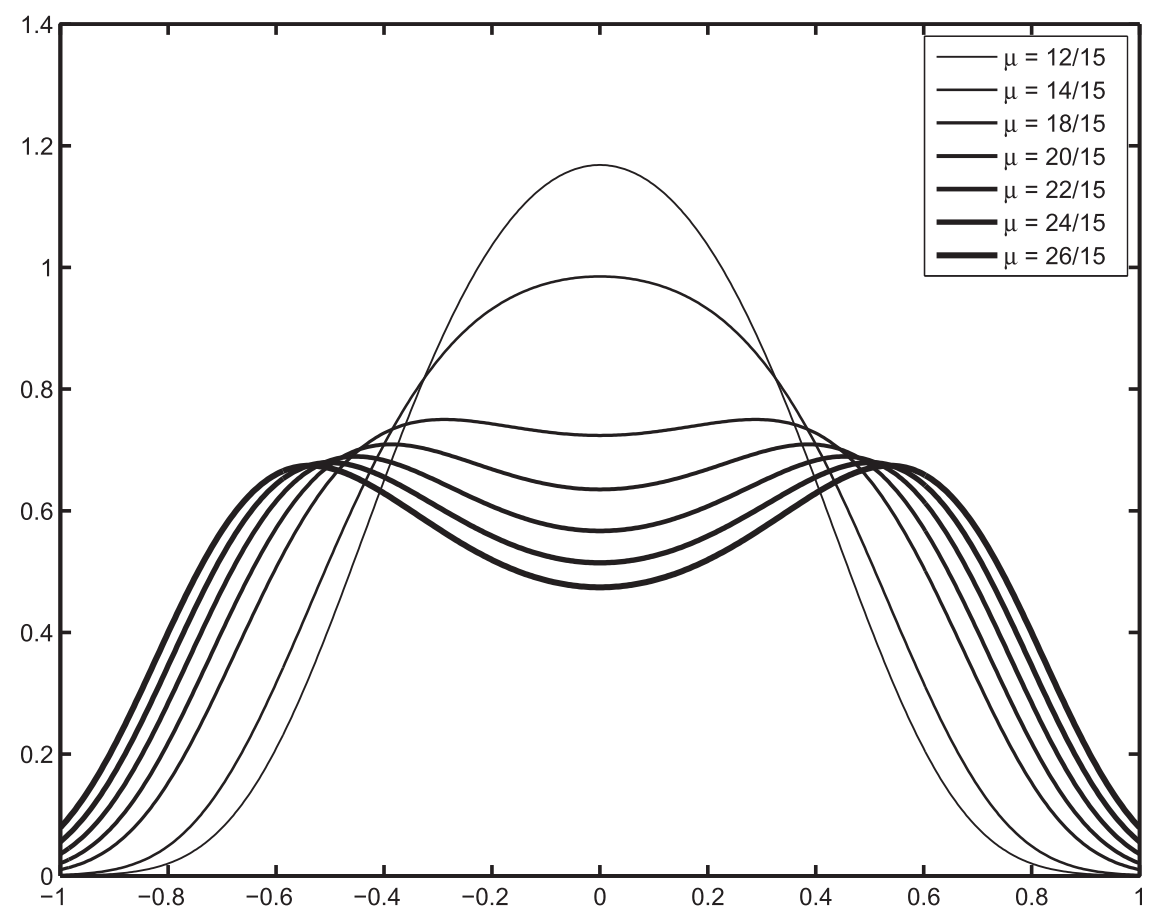

FIG. 12. The stationary solution for the probability distribution for various values of the coupling parameter $\mu$. The solution is unimodal for values below the threshold and becomes bimodal for stronger coupling. It follows closely, of course, the potential distribution in Fig. 9 according to Eq. (20).

identical and the height of the central barrier also increases with increasing values of the coupling.

The solution for the Fokker-Planck equation is a transition probability density distribution that can be expressed in terms of eigenfunctions. The eigenfunctions of the transformed operator $L$ are a complete set and they allow the representation of any initial probability but because of the positive definite nature of the probability every initial condition will always be a mixture of different eigenfunctions. The first five eigenfunctions transformed back using (12) to the eigenfunction of $L_{\mathrm{FP}}$ are shown in Fig. 10. They basically represent an ascending order of orthogonal functions sampling the region around the potential. Except for the mode corresponding to the eigenvalue zero (i.e., the stationary solution), they all have an oscillatory character. The eigenfunctions for the asymmetric case, to be discussed later, is shown in Fig. 11.

It is important to note here that the solution cannot collapse to a single oscillatory eigenvector because only combinations of eigenvectors that preserve the positive definiteness of the probability distribution will be described by the equation.

The different stationary solution obtained for various values of $\mu$ are shown in Fig. 12. The probability has a single maximum for values below the threshold where there is only one critical point and an oscillation, but they become a doublet when $\mu$ is large. The dependence from the value of the noise, $Q$, can be easily guessed from (20): large values of the noise will tend to flatten and eliminate the double peaks, resulting in a single normal distribution centered at the origin. The stationary density probability distribution from the one-dimensional model is therefore very consistent with the probability distribution obtained from the numerical experiments.

The evolution of the probability density distribution can be obtained from the transition probability (18) as

$$
W(x, t)=\int P\left(x, t \mid x^{\prime}, 0\right) W_{\mathrm{IC}}\left(x^{\prime}, 0\right) d x^{\prime}
$$

Figure 13 shows the evolution of the probability for an initial distribution centered at $x=-0.7$. The coupling is set at $\mu=21 / 15$ and it generates a rather well-defined potential with a high barrier. For this value of the noise $(Q=0.15)$ the stationary solution is still not fully achieved at a final time of 60 months.

Several factors can influence the evolution for a given value of the coupling coefficient. Increasing the strength of the forcing will make easier the transition to the neighboring potential well, resulting in a faster convergence to the stationary state. Localization of the initial 


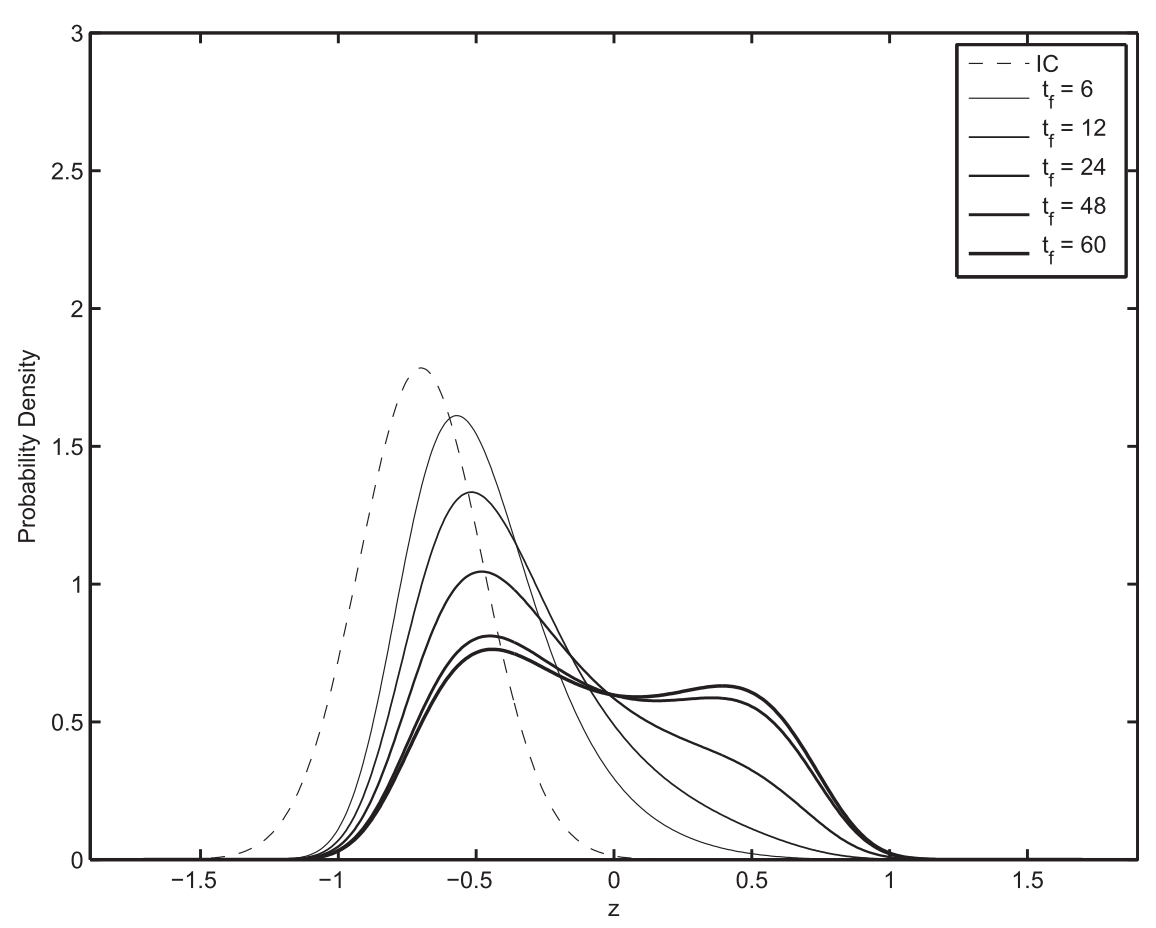

FIG. 13. The evolution of the probability distribution for an initial distribution centered at $x=-0.7$ and for different times in months. The value of the coupling constant used here $(\mu=$ $21 / 15)$ and the level of the stochastic forcing $(Q=0.15)$ result in a time to achieve the stationary distribution of about 60 months.

condition also affects the following evolution. In general, a well-localized distribution will have a slower transition outside of the region where the stationary solution exists, like those in Fig. 13 will have a slower evolution. An extremely localized distribution, as a $\delta(x)$ function, will project equally on all eigenfunctions and the convergence to the zero eigenvalue will be influenced by higher eigenvalues with long decay scales. On the other hand, an initial condition that projects better on the slower mode will have a faster evolution (Fig. 14), showing a rapid convergence to the equilibrium distribution.

\section{The origin of the asymmetry}

The model shown in the previous section shows some of the aspects of the El Niño variability, but it lacks the difference between cold and warm states that the observations have (Fig. 1). An et al. (2005) and An and Jin (2004) have proposed that the effects of the neglected nonlinear terms in the advection can explain some of the amplified magnitude that warm events show. Intraseasonal variations in convection has also received considerable attention for the forcing that they can exert, once their effects is rectified in the seasonal mean, to the interannual variability.
The Madden-Julian oscillation in particular has been the subject of observational and modeling studies. In general, it has been considered to be a component of the stochastic forcing; however, it does seem to have a systematic, rather than stochastic, effects on the anomaly SST in the eastern Pacific. Zhang and Gottschalck (2002) and Zhang (2001) have shown that it is the seasonal activity of the MJO that is correlated with positive SST anomalies in the east Pacific. These results imply that the rectified effect of the $\mathrm{MJO}$ is rather of one sign, tending to push the system toward warmer states. This systematic effect seems to indicate that it is more reasonable to extract the MJO from the stochastic forcing and try to reproduce its effect more directly.

The nonlinear system (39) can then be easily modified to take into account the effect of the MJO by adding a constant term in the temperature equation. However, this would not be consistent with the dynamics of the MJO perturbation on the equatorial Pacific Ocean. A simple constant in the temperature equation will force a change in the thermocline depth of opposite sign, as required by the interannual dynamics. To get a constant response it is necessary to add a term also in the thermocline equation in order to represent the change in depth produced at seasonal scales by the MJO forcing. The 


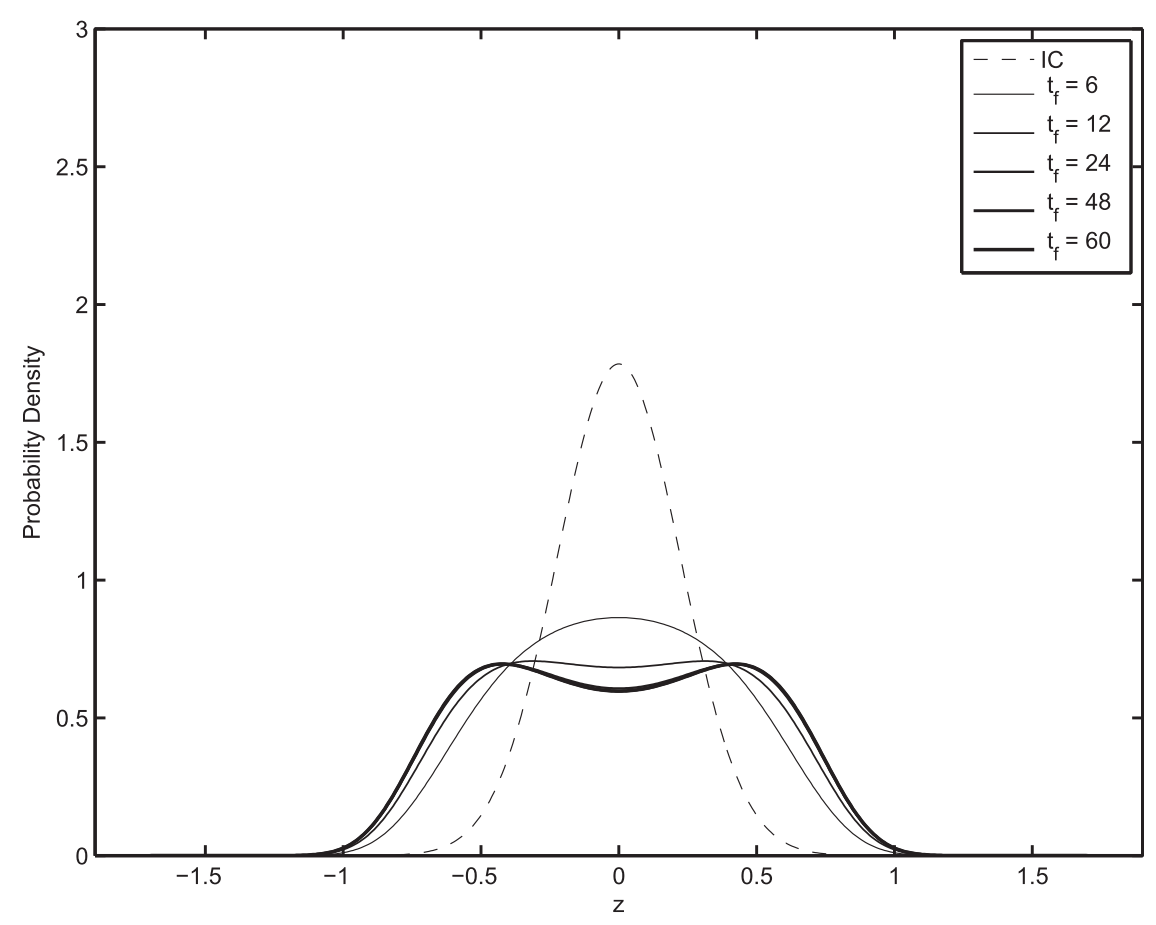

FIG. 14. As in Fig. 13, but for an initial distribution centered at $x=0$.

model Eqs. (39) can therefore be modified adding a constant term $\gamma_{T}$ in the temperature equation and another constant term $\gamma_{h}$ in the thermocline equation such as

$$
\begin{aligned}
& \frac{d h}{d t}=-r h-\alpha \mu b_{0} T+\gamma_{h} \\
& \frac{d T}{d t}=\left(\gamma \mu b_{0}-c\right) T+\gamma h-(b T+h)^{3}+\gamma_{T} .
\end{aligned}
$$

It is still possible to obtain a one-dimensional model such that

$$
\dot{z}=\frac{\left(25 \mu^{2} \gamma_{h}-20 \mu \gamma_{T}\right)}{25 \mu^{2}+16}+z \frac{(15 \mu-16)}{25 \mu^{2}+16}-z^{3} \frac{20 \mu}{25 \mu^{2}+16} .
$$

The scale of the constants can be derived from the observations (Zhang 2001) and it is estimated to be of the order of $0.1^{\circ} \mathrm{C}$ at seasonal scale for the SST and of the order of $2 m$ for the average effect on the thermocline.

The potential that can be obtained using the above constant is shown in Fig. 15. The presence of the MJO breaks the symmetry and makes the potential well for the warm stable case deeper. (The variable $z$ is proportional to the negative of the temperature anomalies.) Increasing the coupling intensifies the asymmetry and the separation between the states, making it more difficult to overcome the central barrier.
Breaking the asymmetry creates a different situation. The initial condition at neutral conditions $(x=0)$ in Fig. 16 is showing a faster adjustment to the equilibrium than the previous case. This is more evident in the initial condition centered at $x=-0.7$ (Fig. 17) that is showing clear signs of a faster transition, even if it is yet not completely finished at 48 months. The inspection of these solutions seems to indicate that breaking the symmetry has changed the structure of the eigenvalues that represent the inverse time scales of the evolution of each eigenfunction.

It is possible to analyze the dependence of the skewness of the theoretical distribution as a function of the coupling parameter $\mu$. Table 1 shows the skewness, computed as the normalized third moment of the distribution, for increasing coupling strength. The value for the observations based on the Niño-3.4 set already used in the paper is also shown at the bottom. The skewness is largely a function of the coupling parameter and of the symmetry parameter $\gamma$, whereas for larger values of the noise it tends to become smaller. Realistic values of skewness can be obtained within the parameter range of the coupling.

\section{a. Time correlations}

Correlations can be easily obtained from the joint probability in the stationary state. The autocorrelation length, for instance, can be written as 


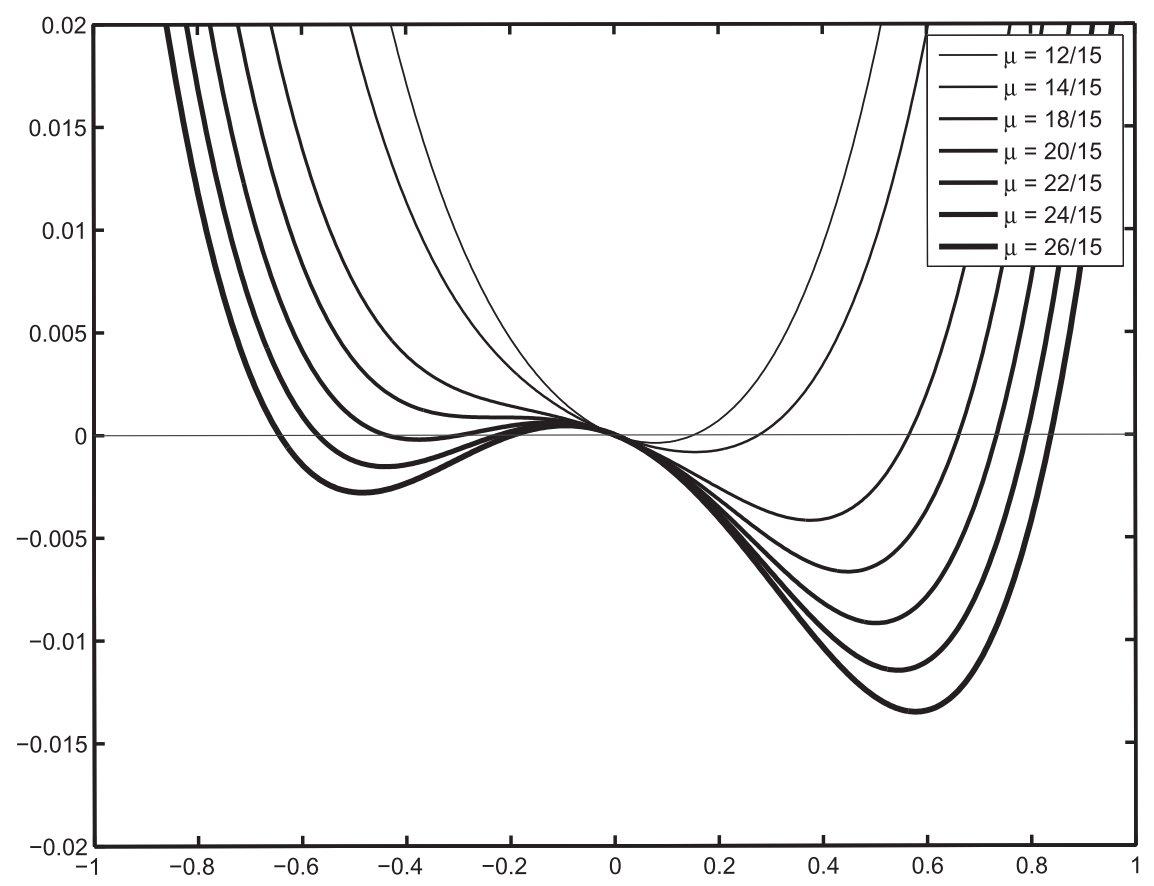

FIG. 15. The potential for the one-dimensional model including the effect of the MJO. The presence of the MJO breaks the symmetry and makes the potential well for the warm stable case deeper. (The variable $z$ is proportional to the negative of the temperature anomalies.) Increasing the coupling intensifies the asymmetry and the separation between the states, making it more difficult to overcome the central barrier.

$$
\begin{aligned}
& \langle x(\tau) x(0)\rangle \\
& =\iint x x^{\prime} e^{\left[U(x)+U\left(x^{\prime}\right)\right] / 2 Q} \sum_{n} \psi_{n}(x) \psi_{n}\left(x^{\prime}\right) e^{-\lambda_{n} \tau} d x d x^{\prime} \\
& \quad \times \iint x x^{\prime} \psi_{0} \psi_{0} \sum_{n} \psi_{n}(x) \psi_{n}\left(x^{\prime}\right) e^{-\lambda_{n} \tau} d x d x^{\prime} .
\end{aligned}
$$

This relation reduces to the covariance for $\tau=0$, whereas the values at different $\tau$ depend on the contribution of each eigenfunction. For very large lags $\tau \rightarrow \infty$ it is interesting to see that we can recover easily the Onsager regression hypothesis so that

$$
\begin{aligned}
& \lim _{\tau \rightarrow \infty}\langle x(\tau) x(0)\rangle \\
& \quad=\iint x x^{\prime} \psi_{0}(x) \psi_{0}\left(x^{\prime}\right) \psi_{0}(x) \psi_{0}\left(x^{\prime}\right) d x d x^{\prime}=\langle x\rangle^{2} .
\end{aligned}
$$

The autocorrelation in general tends to be shorter for increasing noise and increasing asymmetry. The asymmetric potential yields shorter autocorrelations than the symmetric potential for the same value of the coupling and asymmetry parameter $\gamma$. The best results are obtained for relatively large values of gamma; in this case
0.06 was used. Figure 18 shows the autocorrelation for the symmetric and asymmetric potential with $Q=0.1$ and $\mu=27 / 15$. The asymmetric model has some correspondence with the observations for lags up to 10 months, but the symmetric model decays too slowly. The simple asymmetric model captures the main behavior, but it does not fall quickly enough and it does not cross the zero line.

The behavior of the autocorrelation is determined by the eigenvalue structure. Figure 19 shows the structure of the first eigenvalue in the symmetric and in the asymmetric case corresponding to the autocorrelation in Fig. 18. The physical interpretation of these eigenvalues in the quantum mechanics case is that they represent the energy levels of the states; in this case they can be interpreted as the decay time scale for each eigenfunction that contributes to the probability distribution. The symmetric case on the left in Fig. 19 tends to have larger eigenvalues and so the second one has also a very long decay scale of about 38 months inverse. In general the decay scales in the asymmetric case are shorter and so the result is that the autocorrelation as a function of the lag (Fig. 18) is showing a faster drop, on the order of 10 months for the asymmetric case. 


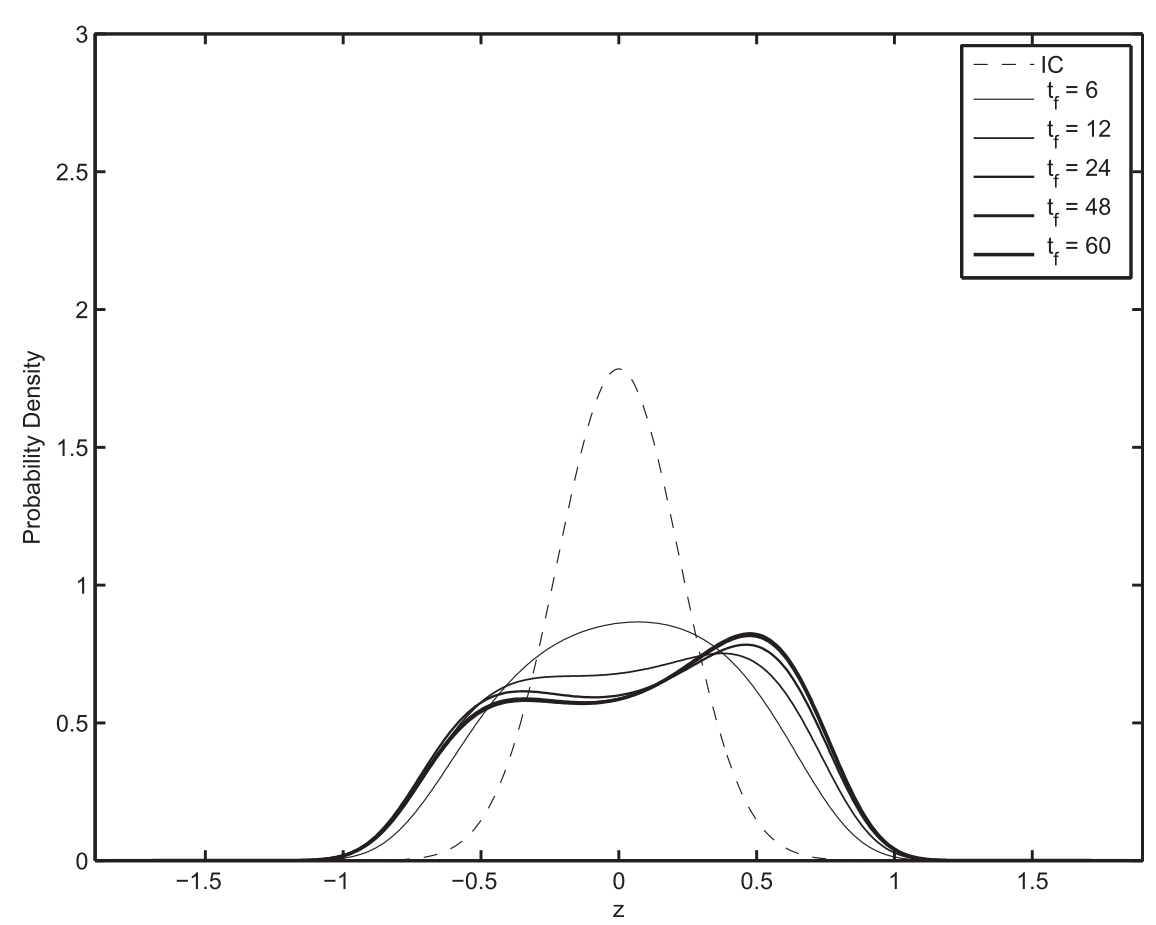

FIG. 16. As in Fig. 14, but for the asymmetric potential.

\section{b. Transition from warm and cold cases}

The probability of transition from one probability distribution to another can be obtained from the density probability distribution [Eqs. (18) or (38)] inserting an initial distribution. In the simplest case we can assume for the initial distribution a delta function in a particular value $W_{\text {IC }}=\delta\left(x^{\prime}-x_{0}\right)$, so that the distribution at time $t$ is given by

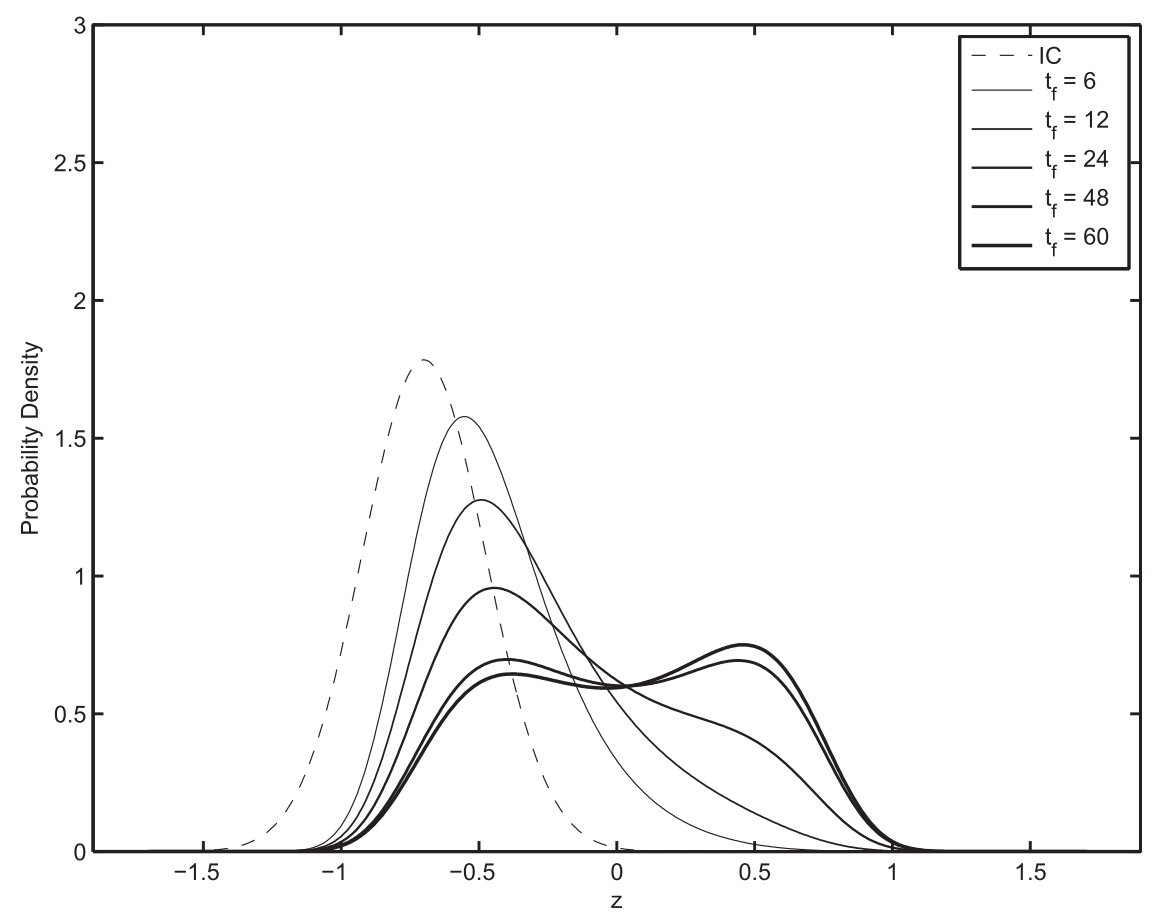

FIG. 17. As in Fig. 13, but for the asymmetric potential. 
TABLE 1. Skewness parameter for the asymmetric distribution and various values of the coupling parameter and for $Q=0.15$ and $\gamma=0.01$. The skewness increases for larger couplings and becomes smaller for increasing noise. The skewness for the estimated probability distribution for the observation shown in Fig. 1 is included at the bottom.

\begin{tabular}{cc}
\hline \hline$\mu$ & Skewness \\
\hline $17 / 15$ & 0.3692 \\
$19 / 15$ & 0.3803 \\
$21 / 15$ & 0.3898 \\
$23 / 15$ & 0.3981 \\
$25 / 15$ & 0.4055 \\
$27 / 15$ & 0.4122 \\
$29 / 15$ & 0.4185 \\
Obs & 0.3633 \\
\hline
\end{tabular}

$$
W(x, t)=\int P\left(x, t \mid x^{\prime}, 0\right) \delta\left(x^{\prime}-x_{0}\right) d x^{\prime}=P\left(x, t \mid x_{0}, 0\right)
$$

It is of particular interest to evaluate the probability to evolve starting from one potential well (a warm El Niño state) to the other well (a cold La Niña state).

In the symmetric case we expect that these two probabilities will be the same because of the symmetry, but the asymmetric case is more interesting. The probability distribution for a transition from a sharp, $\delta$-like distribution at $t=0$ in the cold well located around $z=0.5$ (solid line in Fig. 20) to a later time (in this case 12 months later) is showing a secondary peak in the warm well (located around $z=-0.5$ ), indicating tunneling across the potential barrier induced by the stochastic noise. This is to be expected as we have seen in the previous sections; however, the opposite transition, from the warm well to the cold well (dashed line) is also showing a secondary peak, but the probability density is smaller, indicating that warm states are more likely to be followed by cold states than the opposite. This feature tends to be stronger as the asymmetry increases (growing values of $\mu$ ) or the noise becomes larger. Observations show a similar feature if the probability of the two transitions is estimated from the data (K. Choi and G. Vecchi 2012, unpublished manuscript).

\section{Conclusions}

The probability distribution of the Niño-3.4 index, considered as an indicator of ENSO dynamics, can be reproduced by a simple nonlinear system stochastically forced by Gaussian noise. A probability distribution with many properties similar to the observed Niño-3.4 can be obtained also in a regime that does not support

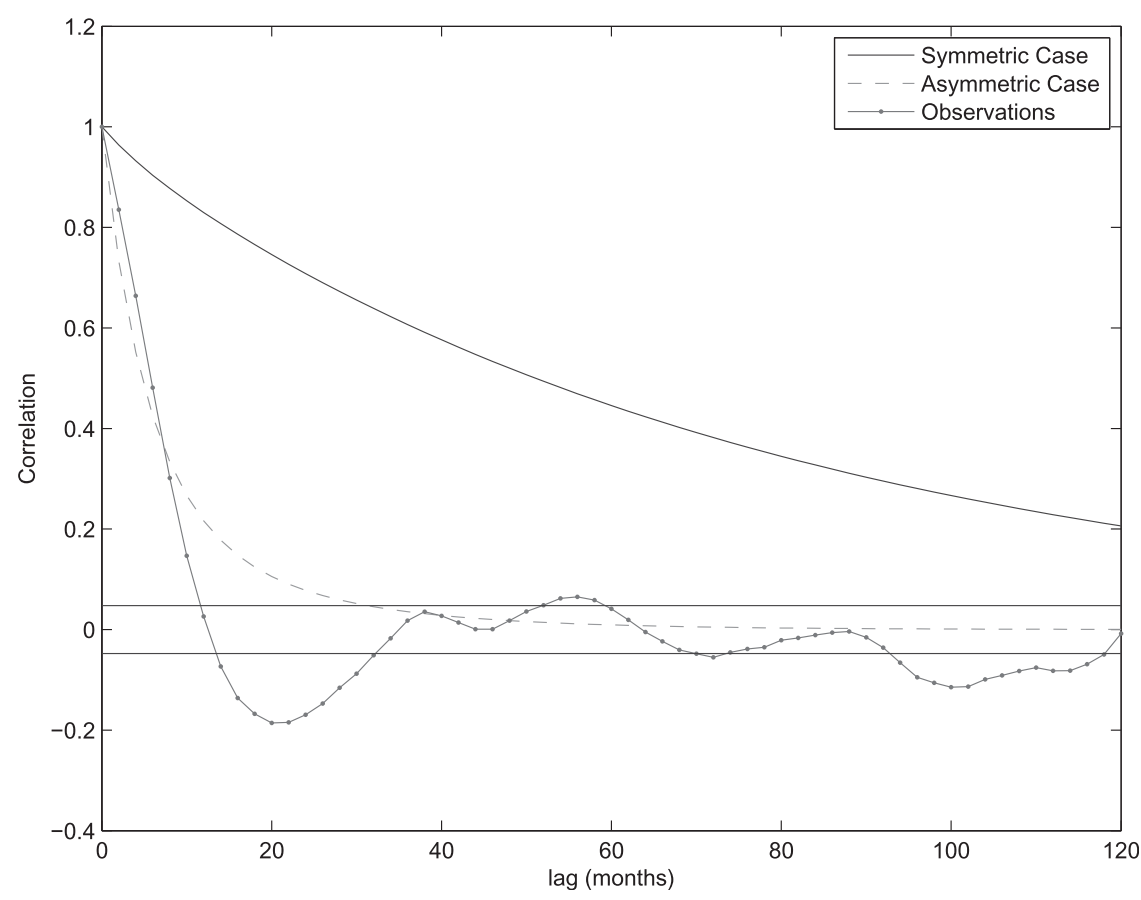

FIG. 18. Autocorrelation function for the two models. The asymmetric model has shorter correlation length for the same value of the coupling constant $\mu$, indicating that the asymmetry is also introducing a faster decorrelation that brings the system to a realistic value of 10-12 months. Horizontal lines indicate the $95 \%$ confidence level for the observed autocorrelations. 


$$
\begin{aligned}
& T_{20}=0.14251 \\
& T_{19}=0.15513 \\
& T_{18}=0.16971 \\
& T_{17}=0.1867 \\
& T_{6}=1.2001 \\
& T_{3}^{2}=5.78 .9222 \\
& T_{7}=0.89267 \\
& T_{16}=0.20668 \\
& T_{10}=0.56156 \\
& T_{12}=0.33773 \\
& T_{15}=0.23046 \\
& T_{14}=0.25911 \\
& T_{13}=0.29415
\end{aligned}
$$

\section{Symmetric Case}

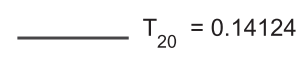

$$
\mathrm{T}_{19}=0.15363
$$$$
\mathrm{T}_{18}=0.16791
$$

$$
\mathrm{T}_{17}=0.18452
$$$$
T_{16}=0.20401
$$$$
T_{15}=0.22714
$$$$
\mathrm{T}_{14}=0.25491
$$$$
\mathrm{T}_{13}=0.28873
$$$$
\mathrm{T}_{12}=0.33058
$$$$
\mathrm{T}_{11}=0.38337
$$$$
\mathrm{T}_{10}=0.45148
$$$$
\mathrm{T}_{9}=0.54181
$$$$
\mathrm{T}_{8}=0.66571
$$$$
\mathrm{T}_{7}=0.843
$$$$
\mathrm{T}_{6}=1.1108
$$$$
\mathrm{T}_{5}=1.5468
$$$$
\mathrm{T}_{4}=2.3182
$$$$
\mathrm{T}_{3}=3.621
$$$$
\mathrm{T}_{2}=9.459
$$$$
T_{1}=\infty
$$

\section{Asymmetric Case}

FIG. 19. The eigenvalues structure for the (left) symmetric and (right) asymmetric models. It is possible to see how breaking the symmetry has increased the separation between the eigenvalues. 


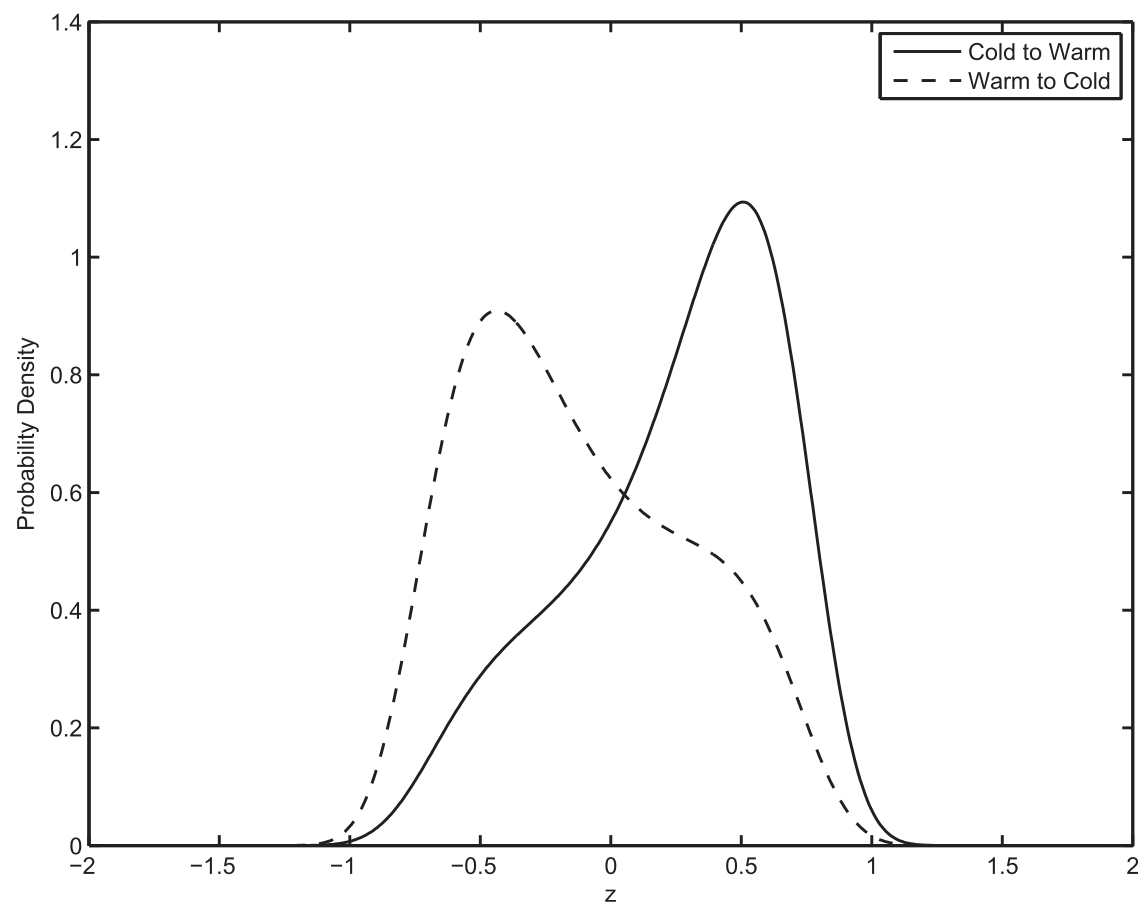

FIG. 20. The probability distribution for a transition from a sharp, $\delta$-like distribution at $t=0$ in the cold well (solid) to $t=12$ months, compared with the same transition from the warm well to the cold well (dashed). The final probability density of the cold to warm transition is smaller than the probability of a warm to cold transition, indicating that El Niño is more likely to be followed by a La Niña than the other way around. The peaks indicate the positions of the cold and warm wells.

self-sustained oscillations, characterized by large values of the coupling constant between stress and surface temperature. This regime has usually been neglected in the past as a regime where ENSO dynamics could not be deployed because of the absence of oscillations, but we show that indeed this may be the case if stochastic forcing is included. The theoretical probability distribution allows the calculation of time correlation and other quantities, showing that the asymmetry is necessary to achieve the time scales that are typical of ENSO. The skewness of the distribution is increasing with the coupling parameter and realistic values can be obtained.

It was not the intention of this paper to advocate a bimodal probability distribution for ENSO; in fact, the distribution is probably unimodal. We propose instead here a representation of ENSO as transitions between states, even as few as only two, that can be a viable framework to study it. Further work is required to clarify what will be the total number of states that will be needed for the final theory.

The Madden-Julian oscillation has a systematic effect on the eastern Pacific SST and on the depth of the thermocline in the western Pacific that can be represented in this model in the simplest way with a constant forcing. The presence of the forcing breaks the symmetry, producing a more realistic asymmetric probability distribution between cold and warm states that also explains the gap in the probability of the warm to cold and cold to warm transition. The theoretical autocorrelation is also impacted by the asymmetries and it tends to be closer to the observations. In this hypothesis, the rectified seasonal effect of the MJO activity has a systematic impact on the ENSO dynamics, producing the asymmetries observed in the probability distribution.

The theory proposed here unifies in a simple logical framework the hypothesis that ENSO is a chaotic nonlinear system or a linear but stochastically forced case; in fact, both these cases can be obtained as limiting cases of the system proposed here. Weak noise and/or weak coupling will result in a self-sustained oscillation, whereas weak nonlinearities (although we did not discuss this case here) will recover the linear stochastic system. This study shows that the dynamics of ENSO is possibly far richer than previously thought and different regimes can be active at different times as the coupling constant and the magnitude of the noise vary. We also think that this approach can be extended in a number of ways, considering 
higher-dimensional systems, multiplicative or colored noise, and more realistic approaches, but this will be the subject of our next papers.

Acknowledgments. The support of the GEMINA project funded by the Ministry for University and Research and by the Ministry of Environment, Land and Sea of Italy is gratefully acknowledged.

\section{REFERENCES}

An, S.-I., and F.-F. Jin, 2004: Nonlinearity and asymmetry of ENSO. J. Climate, 17, 2399-2412.

, Y.-G. Ham, J.-S. Kug, F.-F. Jin, and I.-S. Kang, 2005: El Niño-La Niña asymmetry in the Coupled Model Intercomparison Project simulations. J. Climate, 18, 2617-2627.

Battisti, D. S., and A. C. Hirst, 1989: Interannual variability in a tropical atmosphere-ocean model: Influence of the basic state, ocean geometry, and nonlinearity. J. Atmos. Sci., 46, $1687-1712$.

Berner, J., 2005: Linking nonlinearity and non-Gaussianity of planetary wave behavior by the Fokker-Planck equation. J. Atmos. Sci., 62, 2098-2117.

Brown, J. N., and A. V. Fedorov, 2010: How much energy is transferred from the winds to the thermocline on ENSO time scales? J. Climate, 23, 1563-1580.

Burgers, G., and D. Stephenson, 1999: The "normality" of El Niño. Geophys. Res. Lett., 26, 1027-1030.

Cane, M. A., and S. E. Zebiak, 1985: A theory for El Niño and the Southern Oscillation. Science, 228, 1085-1087.

,-- , and S. C. Dolan, 1986: Experimental forecasts of El Niño. Nature, 321, 827-832, doi:10.1038/321827a0.

Chang, P., L. Ji, H. Li, and M. Flügel, 1996: Chaotic dynamic versus stochastic processes in ENSO in coupled ocean-atmosphere models. Physica D, 98, 301-320.

Fedorov, A. V., S. L. Harper, S. G. Philander, B. Winter, and A. Wittenberg, 2003: How predictable is El Niño? Bull. Amer. Meteor. Soc., 84, 911-919.

Gualdi, S., A. Navarra, and H. von Storch, 1997: Tropical intraseasonal oscillation appearing in operational analyses and in a family of general circulation models. J. Atmos. Sci., 54, 11851202.

_ , E. Guilyardi, P. Delecluse, S. Masina, and A. Navarra, 2003: The role of the Indian Ocean in a coupled model. Climate Dyn., 20, 567-582.

Haken, H., 1976: Generalized Onsager-Machlup function and classes of path integral solutions of the Fokker-Planck equation and the master equation. Z. Phys., 24B, 321-326.

Hendon, H. H., M. C. Wheeler, and C. Zhang, 2007: Seasonal dependence of the MJO-ENSO relationship. J. Climate, 20, 531543.

Jin, F.-F., 1996: Tropical ocean-atmosphere interaction, the Pacific cold tongue, and the El Niño-Southern Oscillation. Science, 274, 76-78, doi:10.1126/science.274.5284.76.

_ 1997: An equatorial ocean recharge paradigm for ENSO. Part I: Conceptual model. J. Atmos. Sci., 54, 811-829.

— atmosphere interaction-A unified view. Part I: Numerical results. J. Atmos. Sci., 50, 3477-3503.

—_ — and M. Ghil, 1994: El Niño on the devil's staircase: Annual subharmonic steps to chaos. Science, 264, 70-72, doi:10.1126/science.264.5155.70.
,-- , and - 1996: El Niño/Southern Oscillation and the annual cycle: Subharmonic frequency locking and aperiodicity. Physica D, 98, 442-465.

- S.-I. An, A. Timmermann, and J. Zhao, 2003: Strong El Niño events and nonlinear dynamical heating. Geophys. Res. Lett., 30, 1120, doi:10.1029/2002GL016356.

Kang, I.-S., and J.-S. Kug, 2002: El Niño-La Niña sea surface temperature anomalies: Asymmetry characteristics associated with their wind stress anomalies. J. Geophys. Res., 107, 4372, doi:10.1029/2001JD000393.

Kapur, A., C. Zhang, J. Zavala-Grey, and H. Hendon, 2012: Role of stochastic forcing in ENSO in observations and a coupled GCM. Climate Dyn., 38, 87-107.

Kleeman, R., 2008: Stochastic theories for the irregularity of the ENSO. Phil. Trans. Roy. Soc., 366A, 2509-2524.

Lengaigne, M., E. Guilyardi, J.-P. Boulanger, C. Menkes, P. Delecluse, P. Inness, J. Cole, and J. Slingo, 2004: Triggering of El Niño by westerly wind events in a coupled general circulation model. Climate Dyn., 23, 601-620, doi:10.1007/ s00382-004-0457-2.

Madden, R. A., and P. R. Julian, 1971: Detection of a 40-50 day oscillation in the zonal wind in the tropical pacific. J. Atmos. Sci., 28, 702-708.

Majda, A., I. Timofeyev, and E. Vanden-Eijnden, 2006: Stochastic models for selected slow variables in large deterministic systems. Nonlinearity, 19, 769-794.

Monahan, A. H., and A. Dai, 2004: The spatial and temporal structure of ENSO nonlinearity. J. Climate, 17, 3026-3036.

Moore, A. M., and R. Kleeman, 1999a: The nonnormal nature of El Niño and intraseasonal variability. J. Climate, 12, 29652982.

— seasonal oscillation. J. Climate, 12, 1199-1220.

Navarra, A., and Coauthors, 2008: Atmospheric horizontal resolution affects tropical climate variability in coupled models. J. Climate, 21, 730-750.

— J. Tribbia, and G. Conti, 2013: The path integral formulation of climate dynamics. Public Libr. Sci. One, 8, e67022, doi:10.1371/journal.pone.0067022.

Neale, R. B., J. H. Richter, and M. Jochum, 2008: The impact of convection on ENSO: From a delayed oscillator to a series of events. J. Climate, 21, 5904-5924.

Neelin, J. D., M. Latif, and F. Jin, 1994: Dynamics of coupled oceanatmosphere models: The tropical problem. Annu. Rev. Fluid Mech., 26, 617-659, doi:10.1146/annurev.fl.26.010194.003153.

—, D. S. Battisti, A. C. Hirst, F.-F. Jin, Y. Wakata, T. Yamagata, and S. E. Zebiak, 1998: ENSO theory. J. Geophys. Res., 103 (C7), 14261-14290.

Penland, C., 1996: A stochastic model of Indo-Pacific sea surface temperature anomalies. Physica D, 98, 534-558.

- 2003: Noise out of chaos and why it won't go away. Bull. Amer. Meteor. Soc., 84, 921-925.

, and P. D. Sardeshmukh, 1995: The optimal growth of tropical sea surface temperature anomalies. J. Climate, 8, 1999-2024.

Philander, S. G., 1990: El Niño, La Niña, and the Southern Oscillation. Academic Press, 293 pp.

— , and A. V. Fedorov, 2003: Is El Niño sporadic or cyclic? Annu. Rev. Earth Planet. Sci., 31, 579-594.

Picaut, J., F. Masia, and Y. du Penhoat, 1997: An advectivereflective conceptual model for the oscillatory nature of the ENSO. Science, 277, 663-666, doi:10.1126/science.277.5326.663.

Rayner, N. A., D. E. Parker, E. B. Horton, C. K. Folland, L. V. Alexander, D. P. Rowell, E. C. Kent, and A. Kaplan, 2003: 
Global analyses of sea surface temperature, sea ice, and night marine air temperature since the late nineteenth century. J. Geophys. Res., 108, 4407, doi:10.1029/2002JD002670.

Risken, H., 1989: The Fokker-Planck Equation. Springer, 472 pp. Schopf, P., and M. J. Suarez, 1988: Vacillations in a coupled oceanatmosphere model. J. Atmos. Sci., 45, 549-566.

Sperber, K., S. Gualdi, S. Legutke, and V. Gayler, 2005: The Madden-Julian oscillation in ECHAM4 coupled and uncoupled general circulation models. Climate Dyn., 25, 117140, doi:10.1007/s00382-005-0026-3.

Suarez, M. J., and P. S. Schopf, 1988: A delayed action oscillator for ENSO. J. Atmos. Sci., 45, 3283-3287.

Subramanian, A. C., M. Jochum, A. J. Miller, R. Murtugudde, R. B. Neale, and D. E. Waliser, 2011: The Madden-Julian oscillation in CCSM4. J. Climate, 24, 6261-6282.

Sura, P., M. Newman, C. Penland, and P. Sardeshmukh, 2005: Multiplicative noise and non-Gaussianity: A paradigm for atmospheric regimes? J. Atmos. Sci., 62, 1391-1409.

Thompson, C. J., and D. S. Battisti, 2000: A linear stochastic dynamical model of ENSO. Part I: Model development. J. Climate, 13, 2818-2832.

, and - 2001: A linear stochastic dynamical model of ENSO. Part II: Analysis. J. Climate, 14, 445-466.
Timmermann, A., F.-F. Jin, and J. Abshagen, 2003: A nonlinear theory for El Niño bursting. J. Atmos. Sci., 60, 152-165.

Tziperman, E., L. Stone, M. A. Cane, and H. Jarosh, 1994: El Niño chaos: Overlapping of resonances between the seasonal cycle and the Pacific Ocean-atmosphere oscillator. Science, 264, 7274, doi:10.1126/science.264.5155.72.

Wang, C., 2001: A unified oscillator model for the El NiñoSouthern Oscillation. J. Climate, 14, 98-115.

Wang, W., and M. J. McPhaden, 2000: The surface-layer heat balance in the equatorial Pacific Ocean. Part II: Interannual variability. J. Phys. Oceanogr., 30, 2989-3008.

Zavala-Garay, J., C. Zhang, A. M. Moore, A. T. Wittenberg, M. J. Harrison, A. Rosati, J. Vialard, and R. Kleeman, 2008: Sensitivity of hybrid ENSO models to unresolved atmospheric variability. J. Climate, 21, 3704-3721.

Zebiak, S. E., and M. A. Cane, 1987: A model El Niño-Southern Oscillation. Mon. Wea. Rev., 115, 2262-2278.

Zhang, C., 2001: Intraseasonal perturbations in sea surface temperatures of the equatorial eastern Pacific and their association with the Madden-Julian oscillation. J. Climate, 14, 1309-1322.

_ Madden-Julian oscillation in the equatorial Pacific. J. Climate, 15, 2429-2445. 